\title{
SECTION PROBLEMS FOR CONFIGURATION SPACES OF SURFACES
}

\author{
LEI CHEN
}

\begin{abstract}
In this paper we give a close-to-sharp answer to the basic questions: When is there a continuous way to add a point to a configuration of $n$ ordered points on a surface $S$ of finite type so that all the points are still distinct? When this is possible, what are all the ways to do it? More precisely, let $\mathrm{PConf}_{n}(S)$ be the space of ordered $n$-tuple of distinct points in $S$. Let $f_{n}(S): \mathrm{PConf}_{n+1}(S) \rightarrow \operatorname{PConf}_{n}(S)$ be the map given by $f_{n}\left(x_{0}, x_{1}, \ldots, x_{n}\right):=\left(x_{1}, \ldots, x_{n}\right)$. We classify all continuous sections of $f_{n}$ up to homotopy by proving the following.

1. If $S=\mathbb{R}^{2}$ and $n>3$, any section of $f_{n}(S)$ is either "adding a point at infinity" or "adding a point near $x_{k}$ ". (We define these two terms in Section 2.1; whether we can define "adding a point near $x_{k}$ " or "adding a point at infinity" depends in a delicate way on properties of $S$. )

2. If $S=S^{2}$ a 2-sphere and $n>4$, any section of $f_{n}(S)$ is "adding a point near $x_{k}$ "; if $S=S^{2}$ and $n=2$, the bundle $f_{n}(S)$ does not have a section. (We define this term in Section 3.2)

3. If $S=S_{g}$ a surface of genus $g>1$ and for $n>1$, we give an easy proof of GG03, Theorem 2] that the bundle $f_{n}(S)$ does not have a section.
\end{abstract}

\section{INTRODUCTION}

Let $M$ be a manifold. There is a natural geometric question: How can we continuously introduce a new point on $M$ for any collection of $n$ distinct points on $M$ ? We denote by $\operatorname{PConf}_{n}(M)$ the pure configuration space parametrizing ordered n-tuple of distinct points on $M$. Let $f_{n}(M)$ : $\mathrm{PConf}_{n+1}(M) \rightarrow \mathrm{PConf}_{n}(M)$ be the map given by $f_{n}\left(x_{0}, x_{1}, \ldots, x_{n}\right):=\left(x_{1}, \ldots, x_{n}\right)$. There is a natural action of permutation group $\Sigma_{n}$ on $\operatorname{PConf}_{n}(M)$ by permuting the $n$ points. Permutation group $\Sigma_{n}$ acts on the fiber bundle $f_{n}(M)$ as well. Thus we get a new fiber bundle $F_{n}(M)$ : $\operatorname{PConf}_{n+1}(M) / \Sigma_{n} \rightarrow \operatorname{PConf}_{n}(M) / \Sigma_{n}$, given by $F_{n}\left(x_{0},\left\{x_{1}, \ldots, x_{n}\right\}\right):=\left\{x_{1}, \ldots, x_{n}\right\}$. The quotient $\operatorname{PConf}_{n}(M) / \Sigma_{n}=: \operatorname{Conf}_{n}(M)$ is called the configuration space parametrizing unordered n-tuple of distinct points on $M$. In this article, we will study the existence and uniqueness of sections of $f_{n}(M)$ and $F_{n}(M)$ when $M$ is a surface.

The study of sections of configuration spaces of open manifolds goes back to the work of McDuff and Segal [Seg74] McD75]. They introduce a point "at infinity", which allows them to prove homological stability for configuration spaces. For closed manifolds, the possibility of adding a point depends on the topology of the manifold. For a manifold $M$ with a nowhere vanishing vector field, Cantero and Palmer [CP15], Berrick, Cohen, Wong and Wu [BCWW06] introduced another way to add a new point by adding a point infinitesimally near an old point using the vector field. This allows Ellenberg and Wiltshire-Gordon [EWG15] to improve eventual polynomiality to immediate polynomiality of the betti numbers of $\operatorname{PConf}_{n}(M)$ for some closed manifolds $M$. The following figures illustrate adding a point "at infinity" and adding a point infinitesimally near an old point on the plane $\mathbb{R}^{2}$. 


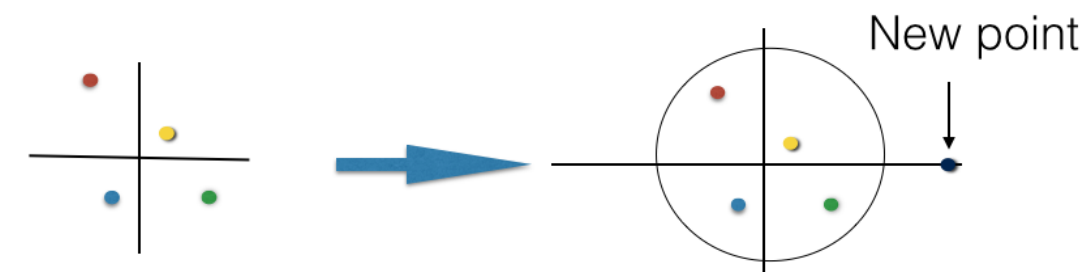

Figure 1.1. "adding a point at infinity"
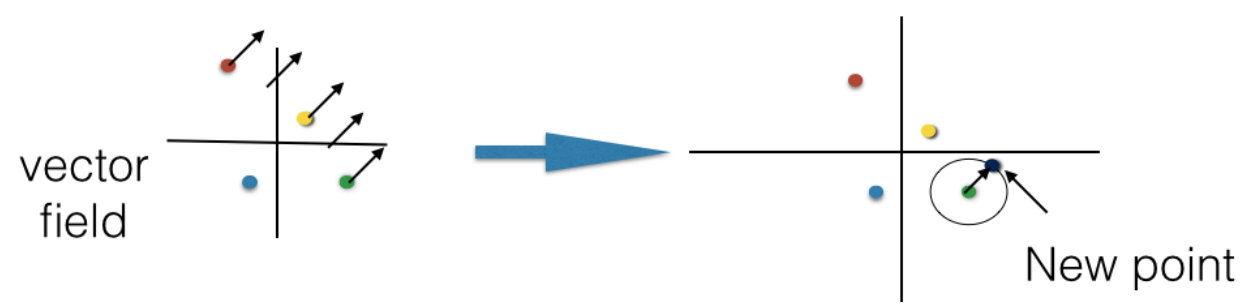

FIGURE 1.2. "adding a point near $x_{k} "$

We call a section $s$ of $f_{n}\left(\mathbb{R}^{2}\right)$ (resp. $f_{n}\left(S^{2}\right)$ ) "adding a point near $x_{k}$ " if $s$ is homotopic to an element in the collection of sections $\operatorname{Add}_{n, k}\left(\mathbb{R}^{2}\right)\left(\operatorname{resp} . \operatorname{Add}_{n, k}\left(S^{2}\right)\right)$. Informally, we assign $x_{0}$ at a sufficiently small distance to $x_{k}$ along some nonvanishing vector field. See Figure 1.2 for a demonstration of "adding a point near $x_{k}$ ". Notice that there are infinitely many homotopy classes of sections in $\operatorname{Add}_{n, k}\left(\mathbb{R}^{2}\right)$ and $\operatorname{Add}_{n, k}\left(S^{2}\right)$ and they are classified by a kind of twists or sections of a circle bundle. See Section 2.1 and Section 3.2 for formal definitions of $\operatorname{Add}_{n, k}\left(\mathbb{R}^{2}\right)$ and $\operatorname{Add}_{n, k}\left(S^{2}\right)$ respectively.

We call a section $s$ of $f_{n}\left(\mathbb{R}^{2}\right)$ "adding a point at infinity" if $s$ is homotopic to an element in the collection of sections $\operatorname{Add}_{n, \infty}\left(\mathbb{R}^{2}\right)$; see Figure 1.1. Informally, we consider $\mathbb{R}^{2}$ as $S^{2}$ missing a point $\infty$, we can assign $x_{0}$ at a sufficiently small distance to $\infty$ along some nonvanishing vector field. See Section 2.1 for a formal definition of $\operatorname{Add}_{n, \infty}\left(\mathbb{R}^{2}\right)$.

Let $S_{g}$ be a surface of genus $g$ and $S^{2}$ be the 2 -sphere. In this paper, we will classify the sections of the fiber bundle $f_{n}(S)$ for 3 cases: $\mathbb{R}^{2}, S^{2}$ and $S_{g}$ when $g>1$. Here by section we mean continuous section.

Theorem 1.1 (Classification of sections for ordered configurations). The following holds:

(1) If $S=\mathbb{R}^{2}$ and $n>3$, any section of $f_{n}(S)$ is either "adding a point at infinity" or "adding a point near $x_{k}$ " for some $1 \leq k \leq n$.

(2) If $S=S^{2}$ and $n=2$, the bundle $f_{n}(S)$ does not have a section. If $S=S^{2}$ and $n>4$, any section of $f_{n}(S)$ is "adding a point near $x_{k}$ " for some $1 \leq k \leq n$.

(3) If $S=S_{g}$ a surface of genus $g>1$ and for $n>1$, the bundle $f_{n}(S)$ does not have a section.

For unordered case, we have the following corollary. 
Corollary 1.2 (Classification of sections for unordered configurations). The following holds:

(1) If $S=\mathbb{R}^{2}$ and $n>3$, any section of $F_{n}(S)$ is "adding a point at infinity";

(2) If $S=S^{2}$ and $n>4$ or $n=2,3$, the bundle $F_{n}(S)$ does not have a section.

Remark 1.3. We discuss the exceptional cases when $n=3$ for $S=S^{2}$ in Section 5.5. Our method does not work for the case $n=4$ but [GG05, Theorem 2] proved that $F_{4}\left(S^{2}\right)$ does not have sections. The $g=1$ case seems to be more complicated to analyze, therefore we do not pursue here. Notice that the construction "adding a point near $x_{k}$ " works for the torus as well; see Section 2.1.

It is classical that $f_{n}\left(\mathbb{R}^{2}\right)$ admits a section. In [Fad62, Theorem 3.1], Fadell showed that when

$n>2$, the bundle $f_{n}\left(S^{2}\right)$ admits a section. The unordered case for $S=\mathbb{R}^{2}$, i.e. (1) of Corollary 1.2 has been proved by [BM06, Main Theorem 2] and [Cas16, Theorem 4]. In [GG05, Theorem 2], they prove the case (2) of Corollary 1.2, and even stronger, they deal with the multi-section problems. All the previous proofs make use of the braid relation and the presentations of braid groups and do not imply (1) and (2) in Theorem 1.1. Our main novelty is to use the characterization of lantern relation in analyzing the canonical reduction systems. The canonical reduction system uses the Thurston classification of isotopy classes of diffeomorphisms of surfaces. This idea originated from BLM83.

The ordered case for $S=S_{g}$ of $g>$ 1, i.e. (3) of Theorem 1.1 has been proved by GG03, Theorem 2]. Their proof makes heavily use of the presentations of surface braid group. We give a simpler proof using the cohomology of surface braid group and a classification theorem in Che16, Theorem 5].

\section{The structure of the paper.}

- In Section 2, we introduce the construction and the main tool we use: canonical reduction system.

- In Section 3, we reduce Theorem 1.1(1) to a more algebraic statement Theorem 3.1.

- In Section 4, we prove Theorem 3.1, which is the main work of this paper.

- In Section 5, we prove Theorem 1.1(2) by reducing it to Theorem 1.1(1).

- In Section 6, we prove Theorem [1.1(3) by a classification of maps between configuration spaces of surfaces in [Che16].

- In Section 7, we ask further questions.

Acknowledgements. The author would like to thank Kevin Casto for telling her about the construction of adding a nearby point and thank Nir Gadish for discussion. She would also like to thank Paolo Bellingeri, Dan Margalit, Cihan Bahran and an anonymous referee for pointing out many references of previous works on braid groups and typos. Finally, she would like to thank her advisor Benson Farb for his extensive comments and for his invaluable support from start to finish. 


\section{The COnstruCtion AND BACKGROUnd on CANONICAL REDUCTION Systems}

Let $S$ be a surface and let $\mathrm{PConf}_{n}(S)$ the pure configuration space be the space of ordered n-tuple of distinct points on $S$. The natural embedding $\operatorname{PConf}_{n}(S) \subset S^{n}$ gives the topology on $\operatorname{PConf}_{n}(S)$. Let $f_{n}(S): \mathrm{PConf}_{n+1}(S) \rightarrow \mathrm{PConf}_{n}(S)$ be the map given by $f_{n}\left(x_{0}, x_{1}, \ldots, x_{n}\right):=\left(x_{1}, \ldots, x_{n}\right)$.

There is a natural action of permutation group $\Sigma_{n}$ on $\operatorname{PConf}_{n}(S)$ by permuting the $n$ points. Thus the quotient space $\operatorname{Conf}_{n}(S)$ is the space of unordered $n$-tuple of distinct points in $S$. Permutation group $\Sigma_{n}$ acts on the fiber bundle $f_{n}(S)$ as well. Let $F_{n}(S): \operatorname{PConf}_{n+1}(S) / \Sigma_{n} \rightarrow$ $\operatorname{PConf}_{n}(S) / \Sigma_{n}$ be the map given by $F_{n}\left(x_{0},\left\{x_{1}, \ldots, x_{n}\right\}\right):=\left\{x_{1}, \ldots, x_{n}\right\}$. The subject of this section is to classify the sections of the fiber bundles $f_{n}\left(\mathbb{R}^{2}\right)$ and $F_{n}\left(\mathbb{R}^{2}\right)$.

2.1. Constructing sections. In this subsection we give constructions of sections of the fiber bundle $f_{n}\left(\mathbb{R}^{2}\right)$. There are two cases: "adding a point near $x_{k}$ " and "adding a point at infinity". These constructions originate from Berrick, Cohen, Wong and Wu [BCWW06], but the idea appeared in FN62.

Case 1: adding a point near $x_{k}$. Define

$\operatorname{PConf}_{n, k}\left(\mathbb{R}^{2}\right)=\left\{\left(v_{k}, x_{1}, \ldots, x_{n}\right) \mid x_{1}, \ldots, x_{n}\right.$ be $n$ points on $\mathbb{R}^{2}$ and $v_{k}$ be a unit vector at $\left.x_{k}\right\}$.

This is the total space of a circle bundle by forgetting the vector $v_{k}$

$$
S^{1} \rightarrow \operatorname{PConf}_{n, k}\left(\mathbb{R}^{2}\right) \rightarrow \operatorname{PConf}_{n}\left(\mathbb{R}^{2}\right) .
$$

Equip $\mathbb{R}^{2}$ with the Euclidean metric. Set

$$
\epsilon\left(x_{1}, \ldots, x_{n}\right)=\frac{1}{2} \min _{1 \leq i \neq j \leq n}\left\{d\left(x_{i}, x_{j}\right)\right\} .
$$

By the definition of $\epsilon\left(x_{1}, \ldots, x_{n}\right)$, setting $x_{0}$ to be the image of the $v_{k}$-flow at time $\epsilon\left(x_{1}, \ldots, x_{n}\right)$ from $x_{k}$ gives a map:

$$
e m_{n, k}\left(\mathbb{R}^{2}\right): \operatorname{PConf}_{n, k}\left(\mathbb{R}^{2}\right) \hookrightarrow \operatorname{PConf}_{n+1}\left(\mathbb{R}^{2}\right) .
$$

Composing a continuous section $s: \operatorname{PConf}_{n}\left(\mathbb{R}^{2}\right) \rightarrow \operatorname{PConf}_{n, k}\left(\mathbb{R}^{2}\right)$ of the fiber bundle 2.1 with $e m_{n, k}\left(\mathbb{R}^{2}\right)$ gives a section of the fiber bundle $f_{n}\left(\mathbb{R}^{2}\right)$.

Definition 2.1 (Adding a point near $\boldsymbol{x}_{\boldsymbol{k}}$ ). We denote by $\operatorname{Add}_{n, k}\left(\mathbb{R}^{2}\right)$ the collection of sections of $f_{n}\left(\mathbb{R}^{2}\right)$ consisting of compositions of a section of 2.1 with $e m_{n, k}\left(\mathbb{R}^{2}\right)$.

Notice that there are infinitely many homotopy classes of sections in $\operatorname{Add}_{n, k}\left(\mathbb{R}^{2}\right)$ and they are in one-to-one correspondence with the homotopy classes of sections of 2.1.

Case 2: adding a point at infinity. Let us call the north pole of a 2 -sphere the point at infinity $\infty$. Then $\mathbb{R}^{2} \cong S^{2}-\infty$ through the stereographic projection. Define

$\operatorname{PConf}_{n, \infty}\left(\mathbb{R}^{2}\right)=\left\{\left(v_{\infty}, x_{1}, \ldots, x_{n}\right) \mid x_{1}, \ldots, x_{n}\right.$ be $n$ points on $\mathbb{R}$ and $v_{\infty}$ be a unit vector at $\left.\infty\right\}$.

This is the total space of a circle bundle by forgetting the vector

$$
S^{1} \rightarrow \operatorname{PConf}_{n, \infty}\left(\mathbb{R}^{2}\right) \rightarrow \operatorname{PConf}_{n}\left(\mathbb{R}^{2}\right)
$$


Equip $S^{2}$ with the spherical metric; i.e. the metric that is induced from the standard embedding $S^{2} \subset \mathbb{R}^{3}$. Set

$$
\epsilon\left(x_{1}, \ldots, x_{n}\right)=\frac{1}{2} \min _{1 \leq i \leq n}\left\{d\left(x_{i}, \infty\right)\right\} .
$$

By the definition of $\epsilon\left(x_{1}, \ldots, x_{n}\right)$, setting $x_{0}$ to be the image of the $v_{\infty}$-flow at time $\epsilon$ from $\infty$ gives a map:

$$
e m_{n, \infty}\left(\mathbb{R}^{2}\right): \operatorname{PConf}_{n, \infty}\left(\mathbb{R}^{2}\right) \hookrightarrow \operatorname{PConf}_{n+1}\left(\mathbb{R}^{2}\right) .
$$

Composing a continuous section $s: \mathrm{PConf}_{n}\left(\mathbb{R}^{2}\right) \rightarrow \operatorname{PConf}_{n, \infty}\left(\mathbb{R}^{2}\right)$ of the fiber bundle 2.2 with $e m_{n, \infty}\left(\mathbb{R}^{2}\right)$ gives a section of the fiber bundle $f_{n}\left(\mathbb{R}^{2}\right)$.

Definition 2.2 (Adding a point at infinity). We denote by $\operatorname{Add}_{n, \infty}\left(\mathbb{R}^{2}\right)$ the collection of sections of $f_{n}\left(\mathbb{R}^{2}\right)$ consisting of compositions of a section of 2.2 with $e m_{n, \infty}\left(\mathbb{R}^{2}\right)$.

Notice that there are infinitely many homotopy classes of sections in $\operatorname{Add}_{n, \infty}\left(\mathbb{R}^{2}\right)$ and they are in one-to-one correspondence with the homotopy classes of sections of 2.2.

2.2. Background. In this subsection we discuss some properties of canonical reduction systems and the lantern relation. Let $S=S_{g, p}^{b}$ be a surface with $b$ boundary components and $p$ punctures. Let $\operatorname{Mod}(S)$ (reps. $\operatorname{PMod}(S)$ ) be the mapping class group (resp. pure mapping class group) of $S$, i.e. the group of isotopy classes of orientation-preserving diffeomorphisms of $S$ fixing the boundary components pointwise and punctures as a set (resp. pointwise). By "simple closed curves", we often mean isotopy class of simple closed curves, e.g. by "preserve a simple closed curve", we mean preserve the isotopy class of a curve.

Thurston's classification of elements of $\operatorname{Mod}(S)$ is a very powerful tool to study mapping class groups. We call a mapping class $f \in \operatorname{Mod}(S)$ reducible if a power of $f$ fixes a nonperipheral simple closed curve. Each nontrivial element $f \in \operatorname{Mod}(S)$ is of exactly one of the following types: periodic, reducible, pseudo-Anosov. See [FM12, Chapter 13] and [FLP12] for more details. We now give the definition of canonical reduction system.

Definition 2.3 (Reduction systems). A reduction system of a reducible mapping class $h$ in $\operatorname{Mod}(S)$ is a set of disjoint nonperipheral curves that $h$ fixes as a set up to isotopy. A reduction system is maximal if it is maximal with respect to inclusion of reduction systems for $h$. The canonical reduction system $\operatorname{CRS}(h)$ is the intersection of all maximal reduction systems of $h$.

For a reducible element $f$, there exists $n$ such that $f^{n}$ fixes each element in $\operatorname{CRS}(f)$ and after cutting out $\operatorname{CRS}(f)$, the restriction of $f^{n}$ on each component is either periodic or pseudo-Anosov. See [FM12, Corollary 13.3]. Now we mention three properties of the canonical reduction systems that will be used later.

Proposition 2.4. $\operatorname{CRS}\left(h^{n}\right)=\mathrm{CRS}(h)$ for any $n$.

Proof. This is classical; see [FM12, Chapter 13].

For a curve $a$ on a surface $S$, denote by $T_{a}$ the Dehn twist about $a$. For two curves $a, b$ on a surface $S$, let $i(a, b)$ be the geometric intersection number of $a$ and $b$. For two sets of curves $P$ and $T$, we say that $S$ and $T$ intersect if there exist $a \in P$ and $b \in T$ such that $i(a, b) \neq 0$. Notice that two sets of curves intersecting does not mean that they have a common element. 
Proposition 2.5. Let $h$ be a reducible mapping class in $\operatorname{Mod}(S)$. If $\{\gamma\}$ and $\operatorname{CRS}(h)$ intersect, then no power of $h$ fixes $\gamma$.

Proof. Suppose that $h^{n}$ fixes $\gamma$. Therefore $\gamma$ belongs to a maximal reduction system $M$. By definition, $\operatorname{CRS}(h) \subset M$. However $\gamma$ intersects some curve in $\operatorname{CRS}(f)$; this contradicts the fact that $M$ is a set of disjoint curves.

Proposition 2.6. Suppose that $h, f \in \operatorname{Mod}(S)$ and $f h=h f$. Then $\operatorname{CRS}(h)$ and $\operatorname{CRS}(f)$ do not intersect.

Proof. By conjugation, we have that $\operatorname{CRS}\left(h f h^{-1}\right)=h(\operatorname{CRS}(f))$. Since $h f h^{-1}=f$, we get that $\operatorname{CRS}(f)=h(\operatorname{CRS}(f))$. Therefore $h$ fixes the whole set CRS $(f)$. A power of $h$ fixes all curves in $\mathrm{CRS}(f)$. By Proposition 2.5, curves in $\mathrm{CRS}(h)$ do not intersect curves in CRS $(f)$.

Now, we introduce a remarkable relation for $\operatorname{Mod}(S)$ that will be used in the proof.

Proposition 2.7 (The lantern relation). There is an orientation-preserving embedding of $S_{0,4} \subset$ $S$ and let $x, y, z, b_{1}, b_{2}, b_{3}, b_{4}$ be simple closed curves in $S_{0,4}$ that are arranged as the curves shown in the following figure.

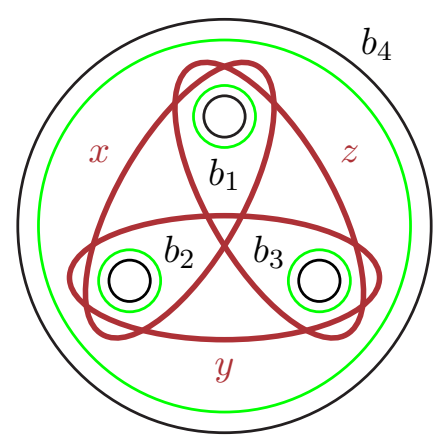

In $\operatorname{Mod}(S)$ we have the relation

$$
T_{x} T_{y} T_{z}=T_{b_{1}} T_{b_{2}} T_{b_{3}} T_{b_{4}}
$$

Proof. This is classical; see [FM12, Chapter 5.1].

\section{An algebraic Result and how it implies (1) of Theorem 1.1}

In this section we give an algebraic result about the braid groups and prove how it implies (1) of Theorem 1.1. $\operatorname{PConf}_{n}\left(\mathbb{R}^{2}\right)$ and $\mathrm{PConf}_{n+1}\left(\mathbb{R}^{2}\right)$ are both $K(\pi, 1)$ spaces. This can be seen by induction on $n$ and taking the long exact sequence of homotopy groups of the fiber bundle $f_{n}\left(\mathbb{R}^{2}\right)$. Therefore, the homotopy classes of sections of $f_{n}\left(\mathbb{R}^{2}\right)$ only depend on the homomorphisms of the fundamental groups. Let $P B_{n}=\pi_{1}\left(\mathrm{PConf}_{n}\left(\mathbb{R}^{2}\right)\right)$ and let $F_{n}$ be a free group of $n$ generators. The fundamental groups of the fiber bundle $f_{n}(S)$ gives us the following short exact sequence, i.e. the Fadell-Neuwirth short exact sequence:

$$
1 \rightarrow F_{n} \rightarrow P B_{n+1} \underset{6}{\stackrel{f_{n}\left(\mathbb{R}^{2}\right)_{*}}{\longrightarrow}} P B_{n} \rightarrow 1
$$


Let $D_{n}$ be the disk with $n$ punctures $\left\{x_{1}, \ldots, x_{n}\right\}$ and $D_{n+1}$ be the disk with $n+1$ punctures $\left\{x_{0}, x_{1}, \ldots, x_{n}\right\}$ and the forget map forgets the point $x_{0}$. We view $P B_{n}$ and $P B_{n+1}$ as mapping class groups as the following:

$$
P B_{n}=\operatorname{PMod}\left(D_{n}\right) \text { and } P B_{n+1}=\operatorname{PMod}\left(D_{n+1}\right) .
$$

A simple closed curve $a$ on $D_{n}$ separates $D_{n}$ into two parts: the outside of a, i.e. the component containing the boundary of $D_{n}$ and the inside of $a$, i.e. the one not containing the boundary of $D_{n}$. We say that a surrounds $x_{k}$ if $x_{k} \in$ the inside of $a$. The following algebraic result on the splittings of the exact sequence (3.1) is a key ingredient in the proof of Theorem 1.1 .

Theorem 3.1. Suppose that we have a section $s: P B_{n} \rightarrow P B_{n+1}$. Then the image $s\left(P B_{n}\right)$ either preserves a simple closed curve $c$ surrounding points $\left\{x_{1}, \ldots, x_{n}\right\}$, or preserves a simple closed curve c surrounding $\left\{x_{i}, x_{0}\right\}$ for some $i \in\{1,2, \ldots, n\}$.

The rest of this subsection focuses on how Theorem 3.1 implies part (1) of Theorem 1.1. Let $c$ be a curve inside $D_{n+1}$ surrounding $k$ points. Let $D_{k}^{l}$ be a disk with $k$ punctures and $l$ open disks removed. We call the boundary of the $l$ disks the small boundary components and the original boundary of $D$ the big boundary component. See the following figure for a geometric explanation.

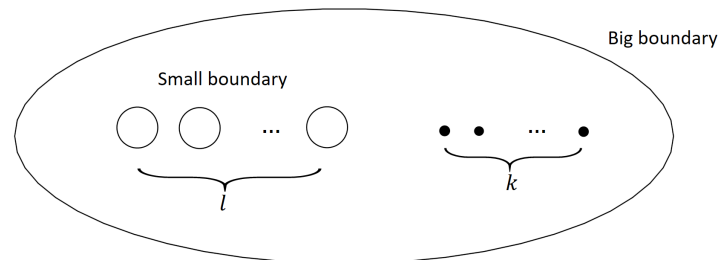

FiguRE 3.1. $D_{k}^{l}$ where small boundaries are the $l$ small circles and big boundary is the outside circle.

Let

$$
P B_{k, l}:=\operatorname{PMod}\left(D_{k}^{l}\right)
$$

be the pure mapping class group of $D_{k}^{l}$. The difference between punctures and boundary components is that the Dehn twist about a puncture is trivial but the Dehn twist about a boundary component is nontrivial. The following proposition describes the centralizer of $T_{c}$. Denote the centralizer of $T_{c}$ by $C_{P B_{n+1}}\left(T_{c}\right)$.

Proposition 3.2 (Centralizer of $\left.\boldsymbol{T}_{\boldsymbol{c}}\right) \cdot C_{P B_{n+1}}\left(T_{c}\right)$ satisfies the following exact sequence

$$
1 \rightarrow \mathbb{Z} \stackrel{\left(T_{c}, T_{c}^{-1}\right)}{\longrightarrow} P B_{k} \times P B_{n+1-k, 1} \rightarrow C_{P B_{n+1}}\left(T_{c}\right) \rightarrow 1
$$

where $k$ is the number of points that $c$ surround.

Proof. This is classical. The centralizer of $T_{c}$ is the subgroup of $\operatorname{Mod}\left(D_{n}\right)$ that fixes $c$. The curve $c$ separates $D_{n}$ into two components: $C_{1}$ that contains the boundary and $C_{2}$ that does not contain the boundary. Since $C_{1}$ and $C_{2}$ are not homeomorphic, we have that $C_{P B_{n+1}}\left(T_{c}\right)$ only contains elements that preserve $C_{1}$ and $C_{2}$. Therefore, our statement holds. 
Now we are ready to prove (1) of Theorem 1.1.

Proof of (1) of Theorem 1.1 assuming Theorem 3.1. Let $g: \operatorname{PConf}_{n}\left(\mathbb{R}^{2}\right) \rightarrow \mathrm{PConf}_{n+1}\left(\mathbb{R}^{2}\right)$ be a section of the fiber bundle $f_{n}\left(\mathbb{R}^{2}\right)$. Let $s=g_{*}: P B_{n} \rightarrow P B_{n+1}$ be the induced map on the fundamental groups of $g$. By Theorem 3.1, the image $s\left(P B_{n}\right)$ preserves a curve $c$ that either surrounds 2 points or $n$ points. Therefore, $s\left(P B_{n}\right)$ is in the centralizer of $T_{c}$ in $P B_{n+1}$ by the fact that $f T_{c} f^{-1}=T_{f(c)}$.

Case 1: when $c$ surrounds $\left\{\boldsymbol{x}_{\mathbf{0}}, \boldsymbol{x}_{\boldsymbol{k}}\right\} . P B_{2} \cong \mathbb{Z}$, which is generated by the Dehn twist about the boundary component. From Proposition 3.2 we have

$$
1 \rightarrow \mathbb{Z} \stackrel{\left(T_{c}, T_{c}^{-1}\right)}{\longrightarrow} \mathbb{Z} \times P B_{n-1,1} \rightarrow C_{P B_{n+1}}\left(T_{c}\right) \rightarrow 1
$$

Therefore $C_{P B_{n+1}}\left(T_{c}\right) \cong P B_{n-1,1}$. The inclusion $P B_{n-1,1} \hookrightarrow P B_{n+1}$ is induced by gluing a 2punctured disk inside the small boundary of $D_{n-1}^{1}$.

On the other hand, we have that

$$
\pi_{1}\left(\mathrm{PConf}_{n, k}\left(\mathbb{R}^{2}\right)\right)=P B_{n-1,1} .
$$

The fundamental groups of the fiber bundle (2.1) is the following exact sequence:

$$
1 \rightarrow \mathbb{Z} \stackrel{T_{d}}{\rightarrow} P B_{n-1,1} \rightarrow P B_{n} \rightarrow 1
$$

Here $T_{d}$ is the Dehn twist about the small boundary component. The embedding $e m_{n, k}: \operatorname{PConf}_{n, k}\left(\mathbb{R}^{2}\right) \hookrightarrow$ $\mathrm{PConf}_{n+1}\left(\mathbb{R}^{2}\right)$ induces a homomorphism on the fundamental group $e m_{n, k *}: P B_{n-1,1} \rightarrow P B_{n+1}$. On the mapping class group level, since $T_{d}$ in $P B_{n-1,1}$ is mapped to the Dehn twist about a curve surrounding $\left\{x_{0}, x_{k}\right\}$, we know that $e m_{n, k *}$ is also induced by gluing a 2-punctured disk inside the small boundary of $D_{n-1}^{1}$. The theorem holds.

Case 2: when $\boldsymbol{c}$ surrounds $\left\{\boldsymbol{x}_{1}, \ldots, \boldsymbol{x}_{\boldsymbol{n}}\right\}$. Since $P B_{1,1} \cong \mathbb{Z} \times \mathbb{Z}$, which is generated by the Dehn twists about the two boundaries, we have the following exact sequence:

$$
1 \rightarrow \mathbb{Z} \stackrel{\left(0, T_{c}, T_{c}^{-1}\right)}{\longrightarrow} \mathbb{Z} \times \mathbb{Z} \times P B_{n} \rightarrow C_{P B_{n+1}}\left(T_{c}\right) \rightarrow 1
$$

On the mapping class group level, $P B_{n} \times P B_{1,1} \rightarrow C_{P B_{n+1}}\left(T_{c}\right) \rightarrow P B_{n+1}$ is induced by gluing $D_{1}^{1}$ outside the big boundary component of $D_{n}$. Therefore $\mathbb{Z} \times P B_{n} \cong C_{P B_{n+1}}\left(T_{c}\right)$ and the generator of $\mathbb{Z}$ is mapped to $T_{c} T_{b}^{-1}$ where $b$ is the big boundary of $D_{n+1}$.

On the other hand, we have that

$$
\pi_{1}\left(\mathrm{PConf}_{n, \infty}\left(\mathbb{R}^{2}\right)\right)=\mathbb{Z} \times P B_{n} .
$$

The embedding $e m_{n, \infty}: \operatorname{PConf}_{n, \infty}\left(\mathbb{R}^{2}\right) \hookrightarrow \operatorname{PConf}_{n+1}\left(\mathbb{R}^{2}\right)$ induces $e m_{n, \infty *}: \mathbb{Z} \times P B_{n} \rightarrow P B_{n+1}$ on the fundamental groups. On the level of mapping class groups, since $\mathbb{Z}$ maps to $T_{c} T_{b}^{-1}$, we know that $e m_{n, \infty *}$ is induced by the embedding of $D_{n}$ in $D_{n+1}$ and maps the generator of $\mathbb{Z}$ to $T_{c} T_{b}^{-1}$. Therefore, $e m_{n, \infty *}$ is induced by gluing $D_{1}^{1}$ outside the big boundary component of $D_{n}$ as well. Our theorem holds. 
Remark 3.3. The classification of the sections of the fiber bundle $f_{n}(S)$ is not entirely the same as the classification of the splittings of the exact sequence (3.1). There is an subtlety coming from the choice of base point in the fundamental groups. Therefore, we classify the splittings of the exact sequence (3.1) up to conjugacy. In Theorem 3.1, all the choices of $c$ is coming from a conjugacy by an element $F_{n}$; thus they decide the same sections.

\section{The Proof of Theorem 3.1}

Throughout the section we prove Theorem 3.1, which implies Theorem 1.1(1). The strategy of the proof is the following. We assume that there exists a section $s: P B_{n} \rightarrow P B_{n+1}$, i.e. $f_{n}\left(\mathbb{R}^{2}\right)_{*} \circ s=i d$. The strategy is that we first determine $s\left(T_{a}\right)$ for any simple closed curve $a$ on $D_{n}$. We first prove that the lift $s\left(T_{a}\right)$ is always a multi-twist about at most two curves on $D_{n+1}$; these two curves or one curve are either trivial or isotopic to $a$ after forgetting the point $x_{0}$. This is done by using a result of McCarthy on centralizer of pseudo-Anosov element and lantern relation. We find a generating set of $P B_{n}$ consisting of Dehn twists about curves bounding two points. We then argue depending on whether $s\left(T_{a}\right)$ is a multi-twist on two curves or a single twist. The main tool of this part is Proposition 4.1, characterizing lantern relation that we deduce from Thurston's construction.

4.1. Step 1: constrain the image of $s\left(T_{c}\right)$ for a simple closed curve $c$. The following proposition characterizes intersection number 2 of two curves and will be used many times in the proof.

Proposition 4.1. Let $i(a, b) \neq 0$. Then $T_{a} T_{b}$ is a multitwist if and only if $i(a, b)=2$.

Proof. This result was previously obtained by Margalit [Mar02] and Hamidi-Tehrani [HT02]. We give a different proof using Thurston's construction; see e.g. [FM12, Theorem 14.1]. There is a subspace $T$ of $S$ that $a, b$ fills, i.e. the tubular neighborhood of $a \cup b$. Let $\left\langle T_{a}, T_{b}\right\rangle$ be the group generated by $T_{a}$ and $T_{b}$ in $\operatorname{Mod}(T)$. Thurston's theorem says that when $a, b$ fill, there is a representation $\rho:\left\langle T_{a}, T_{b}\right\rangle \rightarrow \operatorname{PSL}(2, \mathbb{R})$ such that

$$
T_{a} \rightarrow\left[\begin{array}{cc}
1 & -i(a, b) \\
0 & 1
\end{array}\right] \text { and } T_{b} \rightarrow\left[\begin{array}{cc}
1 & 0 \\
i(a, b) & 1
\end{array}\right]
$$

$\rho(h)$ is parabolic if and only if $h$ is reducible on $T$. We know that

$$
\rho\left(T_{a} T_{b}\right)=\left[\begin{array}{cc}
1 & -i(a, b) \\
0 & 1
\end{array}\right]\left[\begin{array}{cc}
1 & 0 \\
i(a, b) & 1
\end{array}\right]=\left[\begin{array}{cc}
1-i(a, b)^{2} & -i(a, b) \\
i(a, b) & 1
\end{array}\right]
$$

Since $\operatorname{Trace}\left(\rho\left(T_{a} T_{b}\right)\right)=2-i(a, b)^{2}$, we know that $T_{a} T_{b}$ is reducible on $T$ if and only if $i(a, b)=2$. By the lantern relation, we know that $T_{a} T_{b}$ is a multitwist when $i(a, b)=2$.

The following lemma determines $s\left(T_{a}\right)$ for any simple closed curve $a$ on $D_{n}$.

Lemma 4.2 (The lift of a Dehn twist). Let a be a simple closed curve on $D_{n}$, then $s\left(T_{a}\right)$ can only be one of the following three cases: 
(1) It can be a Dehn twist $T_{a^{\prime}}$ about a curve $a^{\prime}$ on $D_{n+1}$ such that after forgetting $x_{0}$, we have $a^{\prime}=a$.

(2) It can be a multitwist $T_{a^{\prime}} T_{c}^{m}$ (i.e. a product of twists on disjoint curves) about two curves $a^{\prime}$ and $c$ on $D_{n+1}$ for $m \in \mathbb{Z}$, where $c$ surrounds 2 points $\left\{x_{0}, x_{k}\right\}$ and after forgetting $x_{0}$, we have that $a^{\prime}=a$.

(3) It can be $T_{a^{\prime}}\left(T_{a^{\prime}} T_{a^{\prime \prime}}^{-1}\right)^{n}$, where $a^{\prime}$ and $a^{\prime \prime}$ are disjoint on $D_{n+1}$ such that after forgetting $x_{0}$, we have that $a^{\prime}=a^{\prime \prime}=a$.

Proof. We start with a the proof of the claim: After forgetting $x_{0}$, any element of $\operatorname{CRS}\left(s\left(T_{a}\right)\right)$ is either $a$ or surrounding one puncture (trivial).

The centralizer of $T_{a}$ contains $T_{b}$ when $a, b$ are disjoint curves. By injectivity of $s$, the centralizer of $s\left(T_{a}\right)$ contains a copy of $\mathbb{Z}^{2}$ when $n>3$. By [McC82, Theorem 1] that the centralizer of a pseudoAnosov element is virtually cyclic, we know that $s\left(T_{a}\right)$ is not pseudo-Anosov. The injectivity of $s$ also implies that $s\left(T_{a}\right)$ is not a torsion element. Therefore we have that $s\left(T_{a}\right)$ is reducible under Thurston's classification of mapping classes. Assume there exists $b^{\prime} \in \operatorname{CRS}\left(s\left(T_{a}\right)\right)$ such that after forgetting $x_{0}$, we have that $b$ is not trivial and $b \neq a$. Since $b^{\prime} \in \operatorname{CRS}\left(s\left(T_{a}\right)\right)$, we have that a power of $s\left(T_{a}\right)$ fixes $b^{\prime}$. Also a power of any mapping class that commutes with $s\left(T_{a}\right)$ fixes $b$ as well. We break our discussion into the following two cases.

- Case 1: If $i(a, b) \neq 0$, then no power of $T_{a}$ fixes $b$. However we also have some power of $s\left(T_{a}\right)$ fixes $b^{\prime}$. This is a contradiction.

- Case 2: If $i(a, b)=0$ but $b \neq a$, then there exists a curve $c$ such that $i(c, b) \neq 0$ but $i(c, a)=0$. Since $s\left(T_{c}\right)$ commutes with $s\left(T_{a}\right)$, we know that $s\left(T_{c}\right)$ preserves $\operatorname{CRS}\left(s\left(T_{a}\right)\right)$. However $i(b, c) \neq 0$, which shows that no power of $T_{c}$ preserve $b$. This contradicts the fact that a power of $s\left(T_{c}\right)$ preserves $b^{\prime}$.

By the disjointness of curves in canonical reduction system, we have that $\operatorname{CRS}\left(s\left(T_{a}\right)\right)$ contains at most 2 curves. We break the rest of the proof into 3 cases.

- Case 1: $\operatorname{CRS}\left(s\left(T_{a}\right)\right)$ only contains one curve $\boldsymbol{a}^{\prime}$. It depends on the location of $x_{0}$, only one side of $a^{\prime}$ will contain $x_{0}$, which means only that side could $s\left(T_{a}\right)$ could possibly be not identity. If $a^{\prime}$ surrounds $x_{0}$ and $a$ surrounds more than 2 points, there is a curve $b$ inside of $a$ containing 2 points, therefore $s\left(T_{a}\right)$ fixes $\operatorname{CRS}\left(s\left(T_{b}\right)\right)$ inside of $a^{\prime}$, which means $s\left(T_{a}\right)$ does not acts as pseudo-Anosov inside $a^{\prime}$. This proves that a power of $s\left(T_{a}\right)$ is the identity on the inside. Therefore a power of $s\left(T_{a}\right)$ is a power of the $T_{a^{\prime}}$. Since $s\left(T_{a}\right)$ is a lift of $T_{a}$, we have that a power of $s\left(T_{a}\right) T_{a^{\prime}}^{-1}$ is the identity. Therefore $s\left(T_{a}\right)=T_{a^{\prime}}$.

If $a^{\prime}$ surrounds $x_{0}$ and $a$ surrounds 2 points, we position $a$ as in the Figure 4.1. 


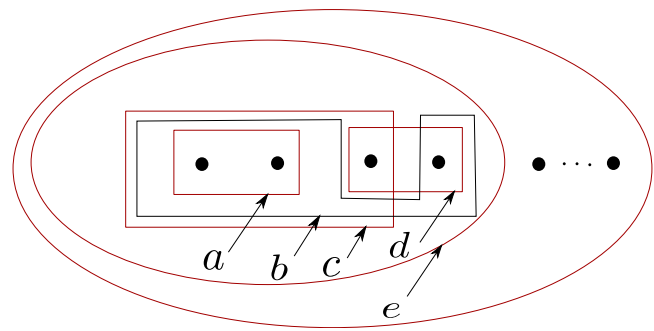

Figure 4.1. $T_{c} T_{d} T_{b}=T_{a} T_{e}$

By the lantern relation, we have

$$
T_{c} T_{d} T_{b}=T_{a} T_{e}
$$

Since $T_{b}, T_{c}, T_{d}, T_{e}$ commutes with $T_{a}$, we have that $s\left(T_{b}\right), s\left(T_{c}\right), s\left(T_{d}\right), s\left(T_{e}\right)$ fix $a^{\prime}$ and therefore is identity on the component that $a^{\prime}$ stay. Since $s\left(T_{a}\right)$ is a product of $s\left(T_{b}\right), s\left(T_{c}\right), s\left(T_{d}\right), s\left(T_{e}\right)$, we know that $s\left(T_{a}\right)$ is also identity in the interior of $a^{\prime}$. Therefore $s\left(T_{a}\right)=T_{a^{\prime}}$. The case when $a^{\prime}$ does not surround $x_{0}$ is similar.

- Case 2: $\operatorname{CRS}\left(s\left(T_{a}\right)\right)$ only contains two curves $a^{\prime}$ and $c$ such that $c$ surrounds 2 points $\left\{\boldsymbol{x}_{\mathbf{0}}, \boldsymbol{x}_{\boldsymbol{k}}\right\}$. On both the exterior of $a^{\prime}$ and the interior of $a^{\prime}$ pinching curve $c$, we know that $s\left(T_{a}\right)$ is identity. Therefore we know that $s\left(T_{a}\right)$ is the multi-twist on $a^{\prime}, c$.

- Case 3: $\operatorname{CRS}\left(s\left(T_{a}\right)\right)$ only contains two curves $a^{\prime}$ and $a^{\prime \prime}$ such that after forgetting $\boldsymbol{x}_{0}$, both curves $\boldsymbol{a}^{\prime}$ and $\boldsymbol{a}^{\prime \prime}$ become $\boldsymbol{a}$. On both the interior of $a^{\prime}$ and the exterior of $a^{\prime \prime}$, we know that $s\left(T_{a}\right)$ is identity. Therefore we know that $s\left(T_{a}\right)$ is the multi-twist on $a^{\prime}, a^{\prime \prime}$.

Notation 4.3. In the following argument, we will use small letters like $a, b, c, \ldots$ to represent simple closed curves on $D_{n}$ and small letters with a prime or double primes like $a^{\prime}, a^{\prime \prime}, b^{\prime}, \ldots$ to represent the canonical reduction systems of $s\left(T_{a}\right), s\left(T_{b}\right), \ldots$ If we have two curves in $\operatorname{CRS}\left(s\left(T_{a}\right)\right)$, we use $a^{\prime}$ and $a^{\prime \prime}$.

4.2. Step 2: the case of adding points at infinity. On $D_{n}$, we call a simple closed curve surrounding 2 points by a basic simple closed curve. The following lemma gives one condition for $s\left(P B_{n}\right)$ to preserve a simple closed curve surrounding $\left\{x_{1}, \ldots, x_{n}\right\}$.

Lemma 4.4. If the canonical reduction system of any basic simple closed curve does not contain a curve surrounding $x_{0}$, then $s\left(P B_{n}\right)$ preserves a simple closed curve surrounding $\left\{x_{1}, \ldots, x_{n}\right\}$.

Proof. Suppose that there exists a simple closed curve $a$ such that $\operatorname{CRS}(a)$ contains a curve surrounding $x_{0}$. We call $a$ the innermost if $a$ surrounds $k$ points and the canonical reduction systems of all curves surrounding $k-1$ points does not contains a curve surrounding $x_{0}$. Take an innermost curve $a$ such that $a$ surrounds $k$ points in $D_{n}$. By the assumption of Lemma 4.4, we have that $k>2$. There are three cases according to Lemma 4.2.

- Case 1: $\operatorname{CRS}(a)=\left\{a^{\prime}\right\}$ such that after forgetting $x_{\mathbf{0}}$, we have $\boldsymbol{a}^{\prime}=\boldsymbol{a}$. We take $b$ and $c$ inside $D_{n}$ as in the following figure, we have the lantern relation $T_{b} T_{c}=T_{e} T_{a} T_{d}^{-1}$. 


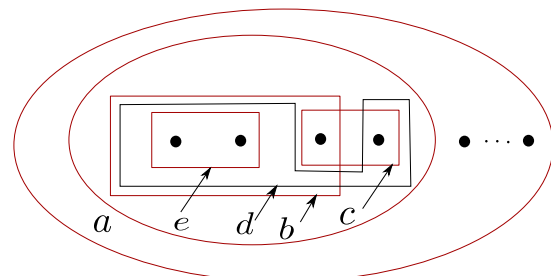

Figure 4.2. $T_{b} T_{c}=T_{e} T_{a} T_{d}^{-1}$

Because $b, c, d$, e surround less points than $k$ and $a$ is the innermost curve, we know that $\operatorname{CRS}\left(s\left(T_{b}\right)\right), \operatorname{CRS}\left(s\left(T_{c}\right)\right), \operatorname{CRS}\left(s\left(T_{d}\right)\right)$ and $\operatorname{CRS}\left(s\left(T_{e}\right)\right)$ each only contains one curve not surrounding $x_{0}$ and we denote them by $b^{\prime}, c^{\prime}, d^{\prime}, e^{\prime}$. Since $T_{e}, T_{a}$ and $T_{d}$ commute with each other, their canonical reduction systems would be disjoint. By Lemma 4.2, we know that $s\left(T_{e} T_{a} T_{d}^{-1}\right)$ is also a multitwist. Therefore by Lemma 4.1. we know that $i\left(b^{\prime}, c^{\prime}\right)=2$. However $\operatorname{CRS}\left(T_{b^{\prime}} T_{c^{\prime}}\right)$ does not contain $a^{\prime}$ because $a^{\prime}$ surround $x_{0}$ but $b^{\prime}, c^{\prime}$ do not. This is a contradiction.

- Case 2: $\operatorname{CRS}(a)=\left\{a^{\prime}, a^{\prime \prime}\right\}$ such that $a^{\prime \prime}$ surrounds 2 points $\left\{x_{0}, x_{k}\right\}$ and after forgetting $x_{0}$, we have that $a^{\prime}=a$.

- Case 3: $\operatorname{CRS}(a)=\left\{a^{\prime}, a^{\prime \prime}\right\}$ such that after forgetting $x_{0}$, we have that $a^{\prime}=$ $a^{\prime \prime}=a$.

Case 2 and 3 can be proved using a similar argument as in Case 1. We construct the same lantern relation and use the fact that $a$ is the innermost curve to reach a contradiction. Therefore if the canonical reduction systems of all basic simple closed curves do not contain a curve surrounding $x_{0}$, then the canonical reduction systems of any curve does not surround $x_{0}$. This is true for the center element of $P B_{n}$ as well. Let $c$ be the boundary curve of $D_{n}$. Then $\operatorname{CRS}(c)=\left\{c^{\prime}\right\}$ does not contain $x_{0}$. However, all Dehn twists commute with $T_{c}$ which preserves $c^{\prime}$.

Now we introduce a generating set for $P B_{n}$. Consider the $n$-punctured disk $D_{n}$ in Figure 4.3 . Let $L$ be a segment below all the other points. Let $L_{1}, \ldots, L_{n}$ be segments connecting $x_{1}, \ldots, x_{n}$ to the segment $L$. Figure 4.4 is the corresponding figure for $D_{n+1}$.

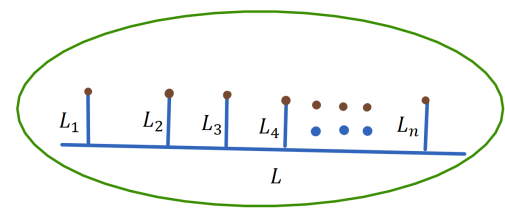

Figure 4.3. $D_{n}$.

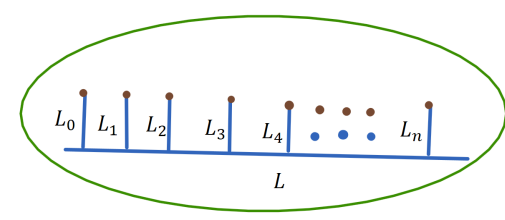

FiguRE 4.4. $D_{n+1}$.

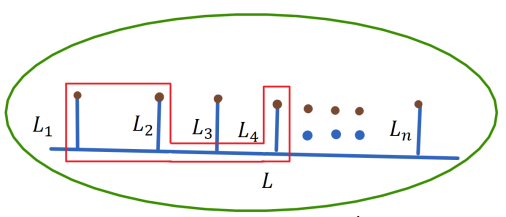

FIGURE 4.5. An example of Notation 4.5 for $a_{124}$. 
Notation 4.5. For $\left\{i_{1}, \ldots, i_{k}\right\}$ a subset of $\{1, \ldots, n\}$, let $a_{i_{1} i_{2} \ldots i_{k}}$ be the boundary curve of the tubular neighborhood of $L \cup \cup \cup_{m=1}^{k} L_{i_{k}}$. Denote by $A_{i_{1} i_{2} \ldots i_{k}}$ the Dehn twist about $a_{i_{1} i_{2} \ldots i_{k}}$. For $\left\{i_{1}, \ldots, i_{k}\right\}$ a subset of $\{0,1, \ldots, n\}$, let $b_{i_{1} i_{2} \ldots i_{k}}$ be the boundary curve of the tubular neighborhood of $L \cup \cup \cup_{m=1}^{k} L_{i_{k}}$. Denote by $B_{i_{1} i_{2} \ldots i_{k}}$ the Dehn twist about $b_{i_{1} i_{2} \ldots i_{k}}$. See Figure 4.5 for an example of a curve representing $a_{124}$.

The following proposition describes a generating set of the group $P B_{n}$.

Proposition 4.6. There is a generating set of $P B_{n}$ consisting of all the Dehn twists about the basic curves $a_{i j}$ for $1 \leq i<j \leq n$.

Proof. This is classical and can be prove it by induction on the exact sequence

$$
1 \rightarrow F_{k} \rightarrow P B_{k+1} \rightarrow P B_{k} \rightarrow 1 \text {. }
$$

This generating set is given by Artin; e.g. see [MM09, Theorem 2.3]

4.3. Step 3: Finishing the proof of Theorem 3.1. In this subsection, we prove Theorem 3.1 . We break the proof into several cases. By Lemma 4.4, we only need to consider the case that there exists at least one basic simple closed curve $a$ such that some element of CRS $(a)$ surrounds $x_{0}$. We break our discussion into the following four cases.

Case 1: The canonical reduction systems of any basic curves only contain one curve $a^{\prime}$ and $a^{\prime}$ surrounds $x_{0}$..

Proof of Case 1. Let $a, b, c, d$ be the curves in Figure 4.6. We have the lantern relation $T_{a} T_{b} T_{c}=T_{d}$.

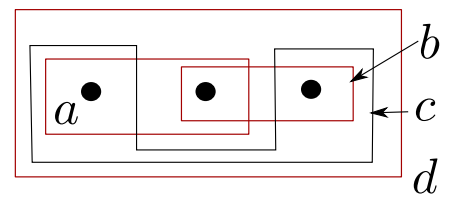

FiguRE 4.6. $D_{n}$

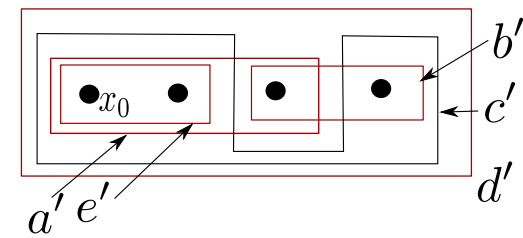

FiguRE $4.7 . D_{n+1}$

Since $T_{c}$ and $T_{d}$ commute, $s\left(T_{c}\right)=T_{c^{\prime}}$ and $s\left(T_{d}\right)=T_{d^{\prime}}$ also commute. Therefore $s\left(T_{a}\right) s\left(T_{b}\right)=$ $T_{c^{\prime}}^{-1} T_{d^{\prime}}$ is a multitwist by Lemma 4.2. By Lemma 4.1, we know that $i\left(b^{\prime}, a^{\prime}\right)=2$ as in Figure 4.7 . Suppose that $b^{\prime}$ does not surround $x_{0}$. By the lantern relation, $T_{a^{\prime}} T_{b^{\prime}}=T_{d^{\prime}} T_{e^{\prime}} T_{c^{\prime}}^{-1}$. Since $s\left(T_{d}\right)$ and $s\left(T_{c}\right)$ are commuting multicurves, $s\left(T_{d}\right) s\left(T_{c}\right)^{-1}$ is multicurve as well. Since $a, b, c$ are basic curves, we know that $s\left(T_{d}\right)=T_{d^{\prime}} T_{e^{\prime}}$ and $s\left(T_{c}\right)=T_{c^{\prime}}$. By the same reason, we have that $s\left(T_{f}\right)=T_{f^{\prime}}$ in Figure 4.8 and 4.9 .

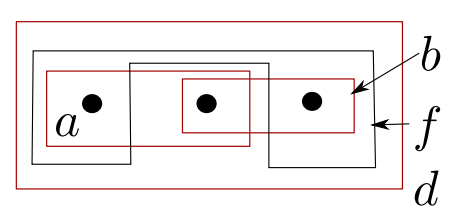

FiguRE 4.8. $D_{n}$

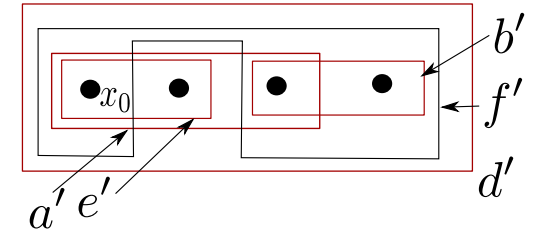

FiguRE 4.9. $D_{n+1}$ 
In the following, we prove that $s\left(P B_{n}\right)$ preserves $b_{01}$. Under Notation 4.5 for $P B_{n}$, we have $a=a_{12}, b=a_{23}, c=a_{13}$ and $d=a_{123}$. We also have that $s\left(A_{12}\right)=B_{012}, s\left(A_{23}\right)=B_{23}$ and $s\left(A_{13}\right)=B_{013}$. Since $A_{i j}$ generates $P B_{n}$, all we need to show is that $s\left(A_{i j}\right)$ preserves $b_{01}$. Since CRS $(d)$ contains $b_{01}$, any curve disjoint from $d$ preserves $b_{01}$. We only need to consider the curves that intersect with $d$. Without loss of generality, we only need to show that $s\left(A_{14}\right), s\left(A_{24}\right)$ and $s\left(A_{34}\right)$ preserve $b_{01}$. By the assumption of Case 1, we only need to show that the $\operatorname{CRS}\left(a_{14}\right)$, $\operatorname{CRS}\left(a_{24}\right)$ and $\operatorname{CRS}\left(a_{34}\right)$ are disjoint from $b_{01}$.

Since $i\left(a_{12}, a_{34}\right)=0$, we have that $\operatorname{CRS}\left(a_{12}\right)$ is disjoint from $\operatorname{CRS}\left(a_{34}\right)$, which means that $\operatorname{CRS}\left(a_{34}\right)$ is disjoint from $b_{01}$. Since $s\left(T_{f}\right)=T_{f^{\prime}}$ in Figure 4.8 and Figure 4.9. $\operatorname{CRS}\left(a_{24}\right)$ is also disjoint from $b_{01}$. We have the following lantern relation:

$$
A_{13} A_{34} A_{14}=A_{134}
$$

The image of relation under lift $s$ is:

$$
B_{013} B_{34} s\left(A_{14}\right)=s\left(A_{134}\right) .
$$

$A_{134}$ commutes with $A_{13}$ and $A_{34}$, thus CRS $\left(a_{134}\right)$ is disjoint from $b_{013}$ and $b_{34}$. The only possible curves are $b_{01}$ and $b_{0134}$. If $s\left(A_{134}\right)=B_{0134}$, we have another lantern relation in $D_{n+1}$ :

$$
B_{013} B_{34} B_{014}=B_{0134} B_{01} .
$$

This proves that $s\left(A_{14}\right)=B_{014} B_{01}^{-1}$ preserving $b_{01}=e^{\prime}$. If $\operatorname{CRS}\left(a_{134}\right)$ contains $b_{01}$, we also have that $s\left(A_{14}\right)$ preserves $b_{01}=e^{\prime}$. The case when $b^{\prime}$ surrounds $x_{0}$ follows from the same argument.

Case 2: There exists a basic simple curve $a$ such that $\operatorname{CRS}(a)$ has two curves and both are isotopic to $a$ after forgetting $x_{0}$.

Proof of Case 2. Let $b, c, d, e$ be curves in Figure 4.10. We have the lantern relation $T_{b} T_{c} T_{d}=T_{e} T_{a}$.

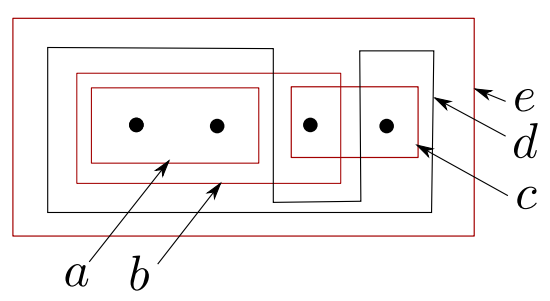

Figure 4.10. $D_{n}$

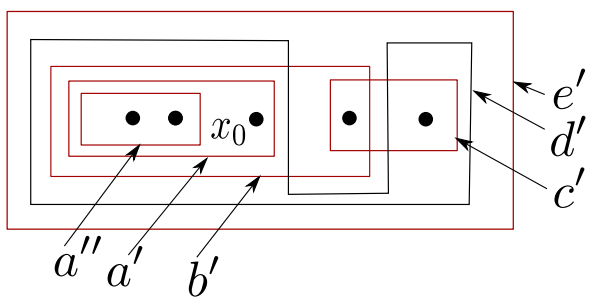

FigURE 4.11. $D_{n+1}$

Since $b, c, d, e$ are disjoint from $a$, we have that $\operatorname{CRS}(b), \operatorname{CRS}(c), \operatorname{CRS}(d)$ and $\operatorname{CRS}(e)$ are disjoint from $\left\{a^{\prime}, a^{\prime \prime}\right\}$. Therefore $s\left(T_{b}\right)=T_{b^{\prime}}, s\left(T_{c}\right)=T_{c^{\prime}}, s\left(T_{d}\right)=T_{d^{\prime}}$ and $s\left(T_{e}\right)=T_{e^{\prime}}$ as in Figure 4.11 . But we also have the lantern relation $T_{b^{\prime}} T_{c^{\prime}} T_{d^{\prime}}=T_{e^{\prime}} T_{a^{\prime}}$. Thus $s\left(T_{a}\right)=T_{a^{\prime}}$. This contradicts the assumption of Case 2 .

Case 3: There exists a basic simple curve $a$ such that CRS $(a)$ has two curves $a^{\prime}, a^{\prime \prime}$ such that $a^{\prime}$ is isotopic to $a$ and $a^{\prime \prime}$ is trivial after forgetting $x_{0}$, and $a^{\prime}$ surrounds $a^{\prime \prime}$. 
Proof of Case 3. We arrange $a$ to be $a_{12}$ and $a^{\prime}, a^{\prime \prime}$ to be $b_{01}, b_{012}$. Then we have $s\left(A_{12}\right)=B_{012} B_{01}^{k}$ for $k \neq 0$ by Lemma 4.2 . Without loss of generality, we only need to show that $\operatorname{CRS}\left(a_{13}\right)$ and $\operatorname{CRS}\left(a_{23}\right)$ are disjoint from $b_{01}$. First of all, we have the following lantern relation:

$$
A_{123} A_{34} A_{124}=A_{12} A_{1234}
$$

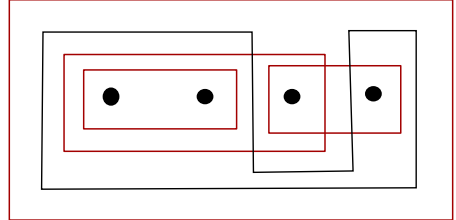

Figure 4.12. $D_{n}$

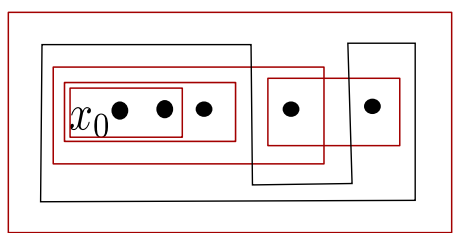

Figure 4.13. $D_{n+1}$

Since all of the curves above are disjoint from $a_{12}$, their canonical reduction systems are disjoint from $a_{12}^{\prime}=b_{01}$ and $a_{12}^{\prime \prime}=b_{012}$. We have the lantern relation:

$$
B_{0123} B_{34} B_{0124}=B_{012} B_{01234} .
$$

Since $s\left(A_{12}\right)=B_{012} B_{01}^{k}$, there exists at least one other curve in $a_{123}, a_{34}, a_{124}, a_{1234}$, whose canonical reduction system contains $b_{01}$. We break our discussion into the following four subcases depending on whether $b_{01}$ is an element in $\operatorname{CRS}\left(A_{1234}\right), \operatorname{CRS}\left(A_{123}\right), \operatorname{CRS}\left(A_{124}\right)$ or $\operatorname{CRS}\left(A_{34}\right)$, respectively.

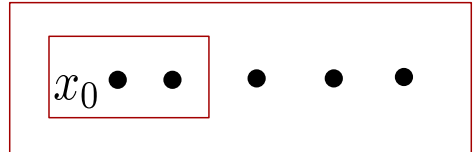

Figure 4.14. $b_{01} \in \operatorname{CRS}\left(A_{1234}\right)$.

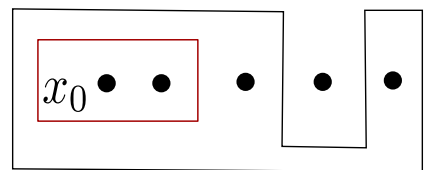

Figure 4.16. $b_{01} \in \operatorname{CRS}\left(A_{124}\right)$.

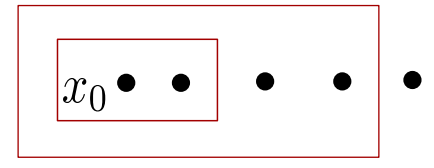

Figure 4.15. $b_{01} \in \operatorname{CRS}\left(A_{123}\right)$.

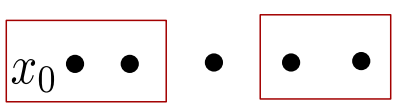

Figure 4.17. $b_{01} \in \operatorname{CRS}\left(A_{34}\right)$.

Subcase 1 and 2: In the first two cases, it is clear that $\operatorname{CRS}\left(a_{13}\right)$ and $\operatorname{CRS}\left(a_{23}\right)$ are disjoint from $b_{01}$ because $a_{13}$ and $a_{23}$ are disjoint from $a_{123}$ and $a_{1234}$.

Subcase 3: By $i\left(a_{14}, a_{124}\right)=0$, we have that $b_{014} \in \operatorname{CRS}\left(a_{14}\right)$ and $b_{01}$ does not intersect $\operatorname{CRS}\left(a_{14}\right)$. Since $i\left(a_{23}, a_{14}\right)=0$, we have that $b_{014}$ does not intersect $\operatorname{CRS}\left(a_{23}\right)$. Suppose $\operatorname{CRS}\left(a_{23}\right)$ contains another curve $z$ that is trivial after forgetting $x_{0}$. Since $a_{23}$ is disjoint from $a_{123}$ and $a_{14}$, we have that $z$ has to be disjoint from $b_{014}$ and $b_{0123}$. The only possibility is that $z=b_{01}$. 
Because of the disjointness of $a_{123}$ and $a_{12}$, we have that $s\left(A_{123}\right)$ preserves $\operatorname{CRS}\left(a_{12}\right)$. This shows that $s\left(A_{123}\right)$ preserves $b_{01}$. We have a lantern relation

$$
A_{12} A_{23} A_{13}=A_{123}
$$

After applying the homomorphism $s$ to the above relation, all of the above element except $s\left(A_{13}\right)$ preserves $b_{01}$. Therefore $s\left(A_{13}\right)$ fixes $b_{01}$.

Subcase 4: $\quad$ Since $i\left(a_{234}, a_{34}\right)=0$, we have that $b_{234} \in \operatorname{CRS}\left(a_{234}\right)$. Since $i\left(a_{123}, a_{12}\right)=0$, we have that $b_{0123} \in \operatorname{CRS}\left(a_{123}\right)$. Therefore $b_{23} \in \operatorname{CRS}\left(a_{23}\right)$ and $\operatorname{CRS}\left(a_{23}\right)$ may contain another curve $z$ that is trivial after forgetting $x_{0}$. However $a_{23}$ is disjoint from $a_{123}$ and $a_{234}$, which implies that $z$ is disjoint from $b_{0123}$ and $b_{234}$. Therefore $z$ can only be $b_{01}$. By the same argument as Subcase 3 , we know that $A_{13}$ also fixes $b_{01}$.

Case 4: There exists a basic simple curve $a$ such that $\operatorname{CRS}(a)$ has two curves $a^{\prime}, a^{\prime \prime}$ such that $a^{\prime}$ is isotopic to $a$ and $a^{\prime \prime}$ is trivial after forgetting $x_{0}$, and $a^{\prime}$ does not surround $a^{\prime \prime}$.

Proof of Case 4. Let $a^{\prime}, a^{\prime \prime}$ be positioned into the following Figure 4.18 such that $a^{\prime}=b_{34}$ and $a^{\prime \prime}=b_{01}$. If a curve $c$ is disjoint from $a_{34}$, then $s\left(T_{c}\right)$ preserves $b_{01}$. Therefore without loss of generality, we only need to show that $s\left(A_{23}\right)$ and $s\left(A_{13}\right)$ preserve $b_{01}$.

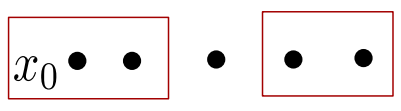

Figure 4.18. $a^{\prime}=b_{34}$ and $a^{\prime \prime}=b_{01}$.

Since $i\left(a_{12}, a_{34}\right)=0$, we have that $b_{012} \in \operatorname{CRS}\left(a_{12}\right)$. Since $i\left(a_{124}, a_{12}\right)=0$, we have that $b_{0124} \in \operatorname{CRS}\left(a_{124}\right)$. Possibly $\operatorname{CRS}\left(a_{124}\right)$ contains another curve $z$ that is trivial after forgetting $x_{0}$. However $b_{24} \in \operatorname{CRS}\left(a_{24}\right)$ because $b_{234} \in \operatorname{CRS}\left(a_{234}\right)$ and $i\left(a_{234}, a_{24}\right)=0$. Therefore, $z$ is disjoint from $b_{24}$ and $b_{012}$, which means $z=b_{01}$. By the same reason, we can prove that $b_{0123} \in \operatorname{CRS}\left(a_{123}\right)$ and $s\left(A_{123}\right)$ preserves $b_{01}$. We have the following lantern relation.

$$
A_{123} A_{34} A_{124}=A_{12} A_{1234}
$$

Since $A_{34}=B_{34} B_{01}^{k}$ for nonzero $k$, therefore the canonical reduction system of one of the curves in the relation $\left(4.2\right.$ contains $b_{01}$. The rest of the discussion is similar to Case 3 by doing a case study.

\subsection{The proof of (1) of Corollary $\mathbf{1 . 2}$,}


Proof of (1) Corollary 1.2. Let $B_{n}=\pi_{1}\left(\mathrm{PConf}_{n}\left(\mathbb{R}^{2}\right) / \Sigma_{n}\right)$ and $B_{n, 1}=\pi_{1}\left(\mathrm{PConf}_{n+1}\left(\mathbb{R}^{2}\right) / \Sigma_{n}\right)$. The fiber bundle $F_{n}(S)$ gives the first line of the following commutative diagram:

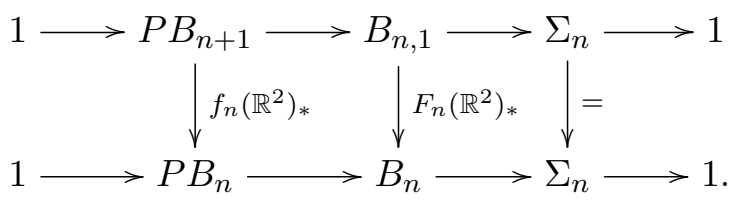

Every splitting of $F_{n}\left(\mathbb{R}^{2}\right)_{*}$ induces a splitting of $f_{n}\left(\mathbb{R}^{2}\right)_{*}$. Therefore, we only need to study the extension of a splitting of $f_{n}\left(\mathbb{R}^{2}\right)_{*}$ to a splitting of $F_{n}\left(\mathbb{R}^{2}\right)_{*}$. Let $\phi: B_{n} \rightarrow B_{n, 1}$ be a splitting of $F_{n}\left(\mathbb{R}^{2}\right)_{*}$. Let $x \in P B_{n}$ and $e \in B_{n}$. We have that

$$
\phi\left(e x e^{-1}\right)=\phi(e) \phi(x) \phi(e)^{-1} .
$$

Denote by $C_{e}$ the conjugation of $e$ on $P B_{n}$. Therefore, we have the following diagram:

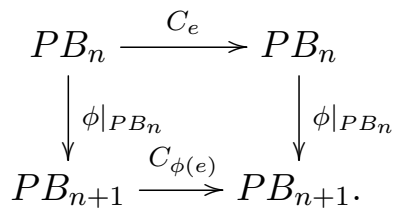

By Theorem 3.1, there are two possibilities of $\left.\phi\right|_{P B_{n}}$ :

(1) $\phi$ fixes a simple closed curve $c$ surrounding $\left\{x_{k}, x_{0}\right\}$

(2) $\phi$ fixes a simple closed curve $c$ surrounding $\left\{x_{1}, \ldots, x_{n}\right\}$.

We claim that $\left.\phi\right|_{P B_{n}}$ fixes a simple closed curve surrounding $\left\{x_{1}, \ldots, x_{n}\right\}$. To prove this claim, we assume the opposite that $\left.\phi\right|_{P B_{n}}$ fixes a simple closed curve $c$ surrounding $\left\{x_{k}, x_{0}\right\}$. There exists an element $e \in B_{n}$ such that $e$ permutes punctures $k$ and $j \neq k$. Since $c$ is the only curve that $\phi\left(P B_{n}\right)$ fixes, we have that $c$ is the only curve that $\phi\left(C_{e}\left(P B_{n}\right)\right)=P B_{n}$ fixes, which contradicts that $C_{\phi(e)}\left(\phi\left(P B_{n}\right)\right)$ also fixes $\phi(e)(c)$ surrounding $\left\{x_{j}, x_{0}\right\}$. Therefore, $\left.\phi\right|_{P B_{n}}$ fixes a simple closed curve surrounds $\left\{x_{1}, \ldots, x_{n}\right\}$. In this case, the section is adding a point at infinity.

\section{The CASE WHEN $S$ IS THE 2-SPhere $S^{2}$}

In this subsection we give a construction of sections of the fiber bundle $f_{n}\left(S^{2}\right)$.

5.1. Nonexistence of a continuous section for $n=2$. We prove a more general result on the sections of the fiber bundle $f_{n}\left(S^{2}\right)$ for $n=2$. Let $S^{2 k}$ be $2 k$-dimensional sphere for $k>0$ integer. Let $x_{1}, x_{2}$ be two distinct points in $S^{2 k}$. The following is classical; see [FH01, Chapter 3].

Proposition 5.1. The following fiber bundle

$$
S^{2 k}-\left\{x_{1}, x_{2}\right\} \rightarrow \operatorname{PConf}_{3}\left(S^{2 k}\right) \stackrel{f_{2}\left(S^{2 k}\right)}{\longrightarrow} \operatorname{PConf}_{2}\left(S^{2 k}\right)
$$

does not have a continuous section. 
Proof. Suppose that there is a continuous map $s: \operatorname{PConf}_{2}\left(S^{2 k}\right) \rightarrow \operatorname{PConf}_{3}\left(S^{2 k}\right)$ such that $f_{2}\left(S^{2 k}\right) \circ$ $s=$ identity. Then after post-composing with a forgetful map to the last coordinate, we obtain a map $f: \operatorname{PConf}_{2}\left(S^{2 k}\right) \rightarrow S^{2 k}$. We denote by $p_{i}: \operatorname{PConf}_{2}\left(S^{2 k}\right) \rightarrow S^{2 k}$ the projection to the $i$ th component. Let

$$
g_{i}: \operatorname{PConf}_{2}\left(S^{2 k}\right) \stackrel{\left(f, p_{i}\right)}{\longrightarrow} \operatorname{PConf}_{2}\left(S^{2 k}\right) \subset S^{2 k} \times S^{2 k} .
$$

Let $\triangle \subset S^{2 k} \times S^{2 k}$ be the diagonal subspace in the product. Let $[\triangle] \in H^{2 k}\left(S^{2 k} \times S^{2 k}, \mathbb{Q}\right)$ be the Poincaré dual of $\triangle$. By the Thom isomorphism, there is an exact sequence for the computation of cohomology:

$$
0 \rightarrow \mathbb{Q} \stackrel{\text { diagonal }}{\longrightarrow} H^{2 k}\left(S^{2 k} \times S^{2 k}, \mathbb{Q}\right) \rightarrow H^{2 k}\left(S^{2 k} \times S^{2 k}-\triangle ; \mathbb{Q}\right) \rightarrow 0 .
$$

Let $c \in H^{2 k}\left(S^{2 k} ; \mathbb{Q}\right)$ be the fundamental class and $c_{i}=p_{i}^{*}(c)$. The image of diagonal is the Thom class $c_{1}+c_{2}$. Therefore in $H^{2 k}\left(S^{2 k} \times S^{2 k}-\triangle ; \mathbb{Q}\right)$, we have $c_{1}+c_{2}=0$. This means that

$$
H^{2 k}\left(S^{2 k} \times S^{2 k}-\triangle ; \mathbb{Q}\right)=\mathbb{Q} c_{1} .
$$

Suppose that $f^{*}(x)=k c_{1}$ for $k$ an integer. Therefore we will have $g_{i}^{*}([\triangle])=k c_{1}+c_{i}$. Since the image of $g_{i}$ misses the diagonal $\triangle$, we have that $g_{1}^{*}([\triangle])=k c_{1}+c_{1}=0$ and $g_{2}^{*}([\triangle])=k c_{1}+c_{2}=$ $k c_{1}-c_{1}=0$. Since $c_{1}$ is a generator of $H^{2 k}\left(S^{2 k} \times S^{2 k}-\triangle ; \mathbb{Q}\right)$, we have that $k+1=0$ and $k-1=0$. These two formulas cannot be satisfied at the same time.

\subsection{Constructing sections when $n>2$. Define}

$\operatorname{PConf}_{n, k}\left(S^{2}\right)=\left\{\left(v_{1}, x_{1}, \ldots, x_{n}\right) \mid x_{1}, \ldots, x_{n}\right.$ be $n$ points in $S^{2}$ and $v_{k}$ be a unit vector at $\left.x_{k}\right\}$.

This is the total space of a circle bundle by forgetting the vector:

$$
S^{1} \rightarrow \operatorname{PConf}_{n, k}\left(S^{2}\right) \rightarrow \operatorname{PConf}_{n}\left(S^{2}\right) .
$$

Proposition 5.2. For $n>2$, the fiber bundle (5.1) is a trivial bundle.

Proof. $S^{1}$-bundle is classified by Euler class, i.e. a second cohomology class of the base. We investigate $H^{2}\left(\operatorname{PConf}_{n}\left(S^{2}\right) ; \mathbb{Z}\right)$ first. There is a graded-commutative $\mathbb{Q}$-algebra $\left[G_{i j}\right]$ defined in [Tot96, Theorem 1], where the degree of the generators $G_{i j}$ is 1 . By Totaro [Tot96, Theorem 1], there is a spectral sequence $E_{2}^{p, q}=H^{p}\left(\left(S^{2}\right)^{n} ; \mathbb{Q}\right)\left[G_{i j}\right]^{q}$ converging to $H^{*}\left(\operatorname{PConf}_{n}\left(S^{2}\right) ; \mathbb{Q}\right)$. Since we only compute $H^{2}$, the differential involved is $d_{2}: E_{2}^{0,1}=H^{0}\left(S^{2} ; \mathbb{Q}\right)\left[G_{i j}\right] \rightarrow E_{2}^{2,0}=H^{2}\left(S^{2} ; \mathbb{Q}\right)$. Let $\left[\triangle_{i j}\right] \in H^{2}\left(S^{2} ; \mathbb{Q}\right)$ be the Poincaré dual of $\triangle_{i j} \subset S^{2}$. By [Tot96, Theorem 2], the differential $d_{2}\left(G_{i j}\right)=\left[\triangle_{i j}\right]$. Let $p_{i}:\left(S^{2}\right)^{n} \rightarrow S^{2}$ be the projection to the $i$ th coordinate and $\left[S^{2}\right] \in H^{2}\left(S^{2} ; \mathbb{Z}\right)$ be the generator of $H^{2}\left(S^{2} ; \mathbb{Z}\right)$. Therefore we have that

$$
H^{2}\left(\operatorname{PConf}_{n}\left(S^{2}\right) ; \mathbb{Z}\right)=\bigoplus_{i=1}^{n} \mathbb{Z} p_{i}^{*}\left[S^{2}\right] /\left(p_{i}^{*}\left[S^{2}\right]+p_{j}^{*}\left[S^{2}\right]\right) \cong \mathbb{Z} / 2,
$$

which is generated by $p_{k}^{*}\left[S^{2}\right]$ and we have that $2 p_{k}^{*}\left[S^{2}\right]=0$. The circle bundle (5.1) is induced from the circle bundle

$$
S^{1} \rightarrow \underset{18}{\mathrm{PConf}_{1,1}\left(S^{2}\right) \rightarrow S^{2}}
$$


by the projection to the $k$ th coordinate. The bundle $(5.2)$ is the unit tangent bundle over $S^{2}$. Since the Euler characteristic of $S^{2}$ is 2 , the Euler class of $(5.2)$ is $e u=2\left[S^{2}\right] \in H^{2}\left(S^{2} ; \mathbb{Z}\right)$. Therefore the Euler class of $[5.1]$ is $p_{k}^{*}[e u]=2 p_{k}^{*}\left[S^{2}\right]=0 \in H^{2}\left(\operatorname{PConf}_{n}\left(S^{2}\right) ; \mathbb{Z}\right)$.

Equip $S^{2}$ with the spherical metric; i.e. the metric that is induced from the standard embedding $S^{2} \subset \mathbb{R}^{3}$. Let

$$
\epsilon\left(x_{1}, \ldots, x_{n}\right)=\frac{1}{2} \min _{1 \leq i<j \leq n}\left\{d\left(x_{i}, x_{j}\right)\right\} .
$$

Set $x_{0}$ to be the image of the $v_{k}$-flow at time $\epsilon$ from $x_{k}$; that is

$$
e m_{n, k}\left(S^{2}\right): \operatorname{PConf}_{n, k}\left(S^{2}\right) \hookrightarrow \operatorname{PConf}_{n+1}\left(S^{2}\right)
$$

Composing a continuous section $s: \operatorname{PConf}_{n}\left(S^{2}\right) \rightarrow \operatorname{PConf}_{n, k}\left(S^{2}\right)$ of the fiber bundle (5.1) with $e m_{n, k}\left(S^{2}\right)$ gives a section of the fiber bundle $f_{n}\left(S^{2}\right)$.

Definition 5.3 (Adding a point near $\boldsymbol{x}_{\boldsymbol{k}}$ ). We denote by $\operatorname{Add}_{n, k}\left(S^{2}\right)$ the collection of sections of $f_{n}\left(S^{2}\right)$ consisting of compositions of a section of (5.1) with $e m_{n, k}\left(S^{2}\right)$.

Notice that there are infinitely many homotopy classes of sections in $\operatorname{Add}_{n, k}\left(S^{2}\right)$ and they are classified by sections of (5.1).

A special section for $\boldsymbol{n}=3$. Since there is a unique Mobius transformation $\phi\left(x_{1}, x_{2}, x_{3}\right)$ that transforms $(0,1, \infty)$ to any ordered three points $\left(x_{1}, x_{2}, x_{3}\right)$. we have that

$$
\operatorname{PConf}_{3}\left(S^{2}\right) \underset{\approx}{\stackrel{\phi}{\Rightarrow}} \operatorname{PSL}(2, \mathbb{C}) .
$$

We can assign any new point $\left.x_{0}=\phi\left(x_{1}, x_{2}, x_{3}\right)\right)(a)$ such that $a \neq 0,1, \infty$.

5.3. The proof of (2) of Theorem 1.1. In this subsection we prove (2) of Theorem 1.1. Let $S_{0, n}$ a sphere with $n$ punctures. Let $\operatorname{Diff}\left(S_{0, n}\right)$ be the orientation-preserving diffeomorphism group of $S_{0, n}$ fixing the $n$ punctures pointwise. While the following is surely known to experts, we could not find this statement or a proof in the literature. I am thus incluing it for completeness. We believe that it follows from Earle-Eells [EE69, Theorem 1] in the punctured case.

Proposition 5.4. For $n>2$, we have that

$$
\operatorname{BDiff}\left(S_{0, n}\right) \cong K\left(\operatorname{PMod}\left(S_{0, n}\right), 1\right)
$$

Proof. We only need to prove that the homotopy group $\pi_{k}\left(\operatorname{Diff}\left(S_{0, n}\right)\right)=0$ for $k>0$. For $n=0$, by Smale [Sma59, Theorem A], $\operatorname{Diff}\left(S^{2}\right) \simeq \mathrm{SO}(3)$. By fiber bundle

$$
\operatorname{Diff}\left(S_{0, n+1}\right) \rightarrow \operatorname{Diff}\left(S_{0, n}\right) \rightarrow S_{0, n}
$$

we deduce that $\operatorname{Diff}\left(S_{0,1}\right) \simeq \mathrm{SO}(2)$ and $\operatorname{Diff}\left(S_{0,2}\right) \simeq \mathrm{SO}(2)$. The long exact sequence of homotopy groups of the fiber bundle $(5.3)$ is

$$
1 \rightarrow \pi_{1}\left(\operatorname{Diff}\left(S_{0,3}\right)\right) \rightarrow \pi_{1}\left(\operatorname{Diff}\left(S_{0,2}\right)\right) \rightarrow \pi_{1}\left(S_{0,2}\right) \rightarrow \operatorname{PMod}_{0,3} \rightarrow \operatorname{PMod}_{0,2} \rightarrow 1 .
$$

However we know that $\operatorname{PMod}_{0,3}=1$ (see [FM12, Proposition 2.3]), we get that $\pi_{1}\left(\operatorname{Diff}\left(S_{0,3}\right)\right)=0$ and also $\pi_{i}\left(\operatorname{Diff}\left(S_{0,3}\right)\right)=0$ for $i>1$. The other cases are the same. 
Let $P B_{n}\left(S^{2}\right)=\pi_{1}\left(\operatorname{PConf}_{n}\left(S^{2}\right)\right)$. Now we are ready to prove $(2)$ of Theorem 1.1 .

Proof of (2) of Theorem 1.1. Let

$$
S_{0, n+1} \rightarrow \operatorname{UDiff}\left(S_{0, n+1}\right) \stackrel{u_{n+1}}{\longrightarrow} \operatorname{BDiff}\left(S_{0, n+1}\right)
$$

be the universal $S_{0, n+1}$-bundle in the sense that any $S^{2}$ bundle with $n+1$ sections

$$
S_{0, n+1} \rightarrow E \rightarrow B
$$

is the pullback from $u_{n+1}$ by a continuous map $f: B \rightarrow \operatorname{BDiff}\left(S_{0, n+1}\right)$. By Proposition 5.4 . $\operatorname{BDiff}\left(S_{0, n+1}\right) \cong K\left(\operatorname{PMod}\left(S_{0, n+1}\right), 1\right)$. This means that $\operatorname{UDiff}\left(S_{0, n+1}\right)$ is also a $K(\pi, 1)$-space. Therefore $S_{0, n+1}$-bundles are determined by their monodromy representations and the sections of an $S_{0, n+1}$-bundle are also determined by the maps on fundamental groups. A splitting of the following exact sequence gives us a section of the fiber bundle $f_{n}\left(S^{2}\right)$.

$$
1 \rightarrow F_{n} \rightarrow P B_{n+2}\left(S^{2}\right) \stackrel{f_{n}\left(S^{2}\right)_{*}}{\longrightarrow} P B_{n+1}\left(S^{2}\right) \rightarrow 1 .
$$

We have the following diagram:

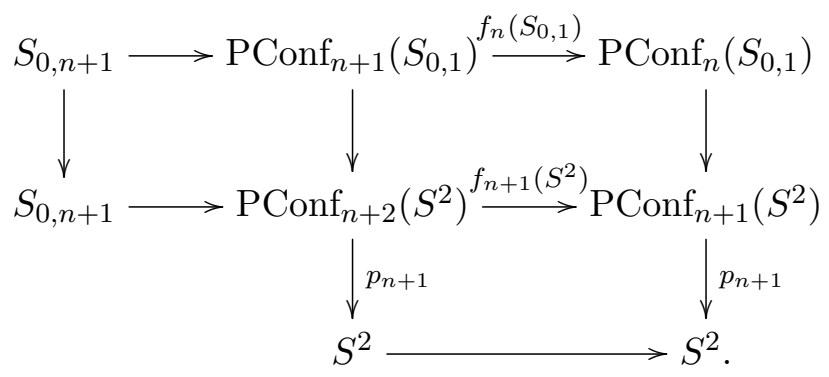

By the long exact sequence of homotopy groups of the fiber bundle

$$
\operatorname{PConf}_{n}\left(S_{0,1}\right) \rightarrow \operatorname{PConf}_{n+1}\left(S^{2}\right) \rightarrow S^{2},
$$

we have that $P B_{n+1}\left(S^{2}\right)=P B_{n} / Z$ where $Z$ denotes the center of $P B_{n}$ and is generated by the Dehn twist about the boundary of $D_{n}$; see [FM12, Page 247]. Therefore a section of $f_{n}\left(S_{0,1}\right)$ induced from a section of $f_{n+1}\left(S^{2}\right)$ satisfies that $f_{n}\left(S_{0,1}\right)_{*}$ maps the center to the center.

Since $S_{0,1} \approx \mathbb{R}^{2}$, the section problem for $f_{n}\left(S_{0,1}\right)$ has been fully discussed in Section 2 . Every section of $f_{n+1}\left(S^{2}\right)$ induces a section of $f_{n}\left(\mathbb{R}^{2}\right)$, thus we could use the classification of sections of $f_{n}\left(\mathbb{R}^{2}\right)$ to study the sections of $f_{n+1}\left(S^{2}\right)$. Let $s: P B_{n+1}\left(S^{2}\right) \rightarrow P B_{n+2}\left(S^{2}\right)$ be a splitting of $f_{n+1}\left(S^{2}\right)_{*}$ such that $f_{n+1}\left(S^{2}\right)_{*} \circ s=i d$. By (1) of Theorem 1.1, we break the discussion into the following two cases according to the sections of $f_{n}\left(S_{0,1}\right)$.

Case 1: the section of $\boldsymbol{f}_{\boldsymbol{n}}\left(\boldsymbol{S}_{\mathbf{0 , 1}}\right)$ is adding a point near $\boldsymbol{x}_{\boldsymbol{k}}$. In this case, $s\left(P B_{n+1}\left(S^{2}\right)\right)$ fixes a curve $c$ around $\left\{x_{0}, x_{k}\right\}$. Then the image lies in the stabilizer of $c$. The stabilizer of $c$ in $\operatorname{PMod}\left(S_{0, n+2}\right)$ is $\operatorname{PMod}\left(D_{n}\right) \cong P B_{n}$. The boundary of $D_{n}$ is $c$ surrounding $\left\{x_{1}, \ldots, x_{k-1}, x_{k+1}, \ldots, x_{n+1}\right\}$. On the other hand by Proposition 5.2 the circle bunlde

$$
S^{1} \rightarrow \operatorname{PConf}_{n, k}\left(S^{2}\right) \rightarrow \operatorname{PConf}_{n}\left(S^{2}\right)
$$


is trivial, we have that

$$
\pi_{1}\left(\mathrm{PConf}_{n+1, k}\left(S^{2}\right)\right) \cong \mathbb{Z} \times \pi_{1}\left(\mathrm{PConf}_{n+1}\left(S^{2}\right)\right) \cong \mathbb{Z} \times P B_{n+1}\left(S^{2}\right) \cong P B_{n} .
$$

The last isomorphism is coming from the splitting of the following exact sequence; see [FM12, Page $252]$.

$$
1 \rightarrow Z \rightarrow P B_{n} \rightarrow P B_{n} / Z \rightarrow 1 \text {. }
$$

Since the $\mathbb{Z}$ component of $\pi_{1}\left(\operatorname{PConf}_{n+1, k}\left(S^{2}\right)\right)$ is mapped to the Dehn twist about a curve $d$ surrounding $\left\{x_{0}, x_{k}\right\}$, it means that $d$ also surrounds $\left\{x_{1}, \ldots, x_{k-1}, x_{k+1}, \ldots, x_{n+1}\right\}$. Therefore we have that $f_{n+1}\left(S^{2}\right)$ is adding a point near $x_{k}$.

Case 2: the section of $\boldsymbol{f}_{\boldsymbol{n}}\left(\boldsymbol{S}_{\mathbf{0}, \mathbf{1}}\right)$ is adding a point near $\boldsymbol{\infty}$. In this case, $s\left(P B_{n+1}\left(S^{2}\right)\right)$ fixes a curve $c$ around $\left\{x_{1}, \ldots, x_{n}\right\}$. Then the image lies in the stabilizer of $c$. The stabilizer of $c$ in $\operatorname{Mod}\left(S_{0, n+2}\right)$ is $\operatorname{PMod}\left(D_{n}\right) \cong P B_{n}$. The boundary of $D_{n}$ is $c$ surrounding $\left\{x_{1}, \ldots, x_{n}\right\}$. On the other hand by Proposition 5.2 the circle bunlde

$$
S^{1} \rightarrow \operatorname{PConf}_{n+1, n+1}\left(S^{2}\right) \rightarrow \operatorname{PConf}_{n+1}\left(S^{2}\right) .
$$

is trivial, we have that

$$
\pi_{1}\left(\operatorname{PConf}_{n+1, n+1}\left(S^{2}\right)\right) \cong \mathbb{Z} \times \pi_{1}\left(\operatorname{PConf}_{n+1}\left(S^{2}\right)\right) \cong \mathbb{Z} \times P B_{n+1}\left(S^{2}\right) \cong P B_{n} .
$$

Since the $\mathbb{Z}$ component of $\pi_{1}\left(\mathrm{PConf}_{n+1, k}\left(S^{2}\right)\right)$ is mapped to the Dehn twist about a curve $d$ surrounding $\left\{x_{0}, x_{n+1}\right\}$, it means that $d$ also surrounds $\left\{x_{1}, \ldots, x_{n}\right\}$. Therefore we have that $f_{n+1}\left(S^{2}\right)$ is adding a point near $x_{n+1}$.

\subsection{The unordered case.}

Proof of (2) of Corollary 1.2. By the same argument as the proof of (1) of Corollary 1.2, we show that none of the sections of

$$
f_{n}\left(S^{2}\right): \operatorname{PConf}_{n+1}\left(S^{2}\right) \rightarrow \operatorname{PConf}_{n}\left(S^{2}\right)
$$

can be extended to a section of

$$
F_{n}\left(S^{2}\right): \operatorname{PConf}_{n+1}\left(S^{2}\right) / \Sigma_{n} \rightarrow \operatorname{PConf}_{n}\left(S^{2}\right) / \Sigma_{n} .
$$

5.5. The exceptional cases. For the special cases $n=3$, we have the following classification.

Theorem 5.5 (Classification of sections of $f_{3}\left(S^{2}\right)$ and $F_{3}\left(S^{2}\right)$ ). There is a unique section for the fiber bundle $f_{3}\left(S^{2}\right)$ up to homotopy. There is no section for the bundle $F_{3}\left(S^{2}\right)$.

Proof. By Proposition 5.4, we have that $\operatorname{BDiff}\left(S_{0,3}\right) \cong K\left(\operatorname{PMod}\left(S_{0,3}\right), 1\right)$. Since $\operatorname{PMod}\left(S_{0,3}\right)=1$, the classifying space $\operatorname{BDiff}\left(S_{0,3}\right)$ is contractible. Therefore every $S^{2}$-bundle with 3 sections is a trivial bundle. Thus $f_{3}\left(S^{2}\right)$ is a trivial bundle. Therefore, a section of $f_{3}\left(S^{2}\right)$ is determined by a map $\operatorname{PConf}_{3}\left(S^{2}\right) \rightarrow S_{0,3}$. Since $S_{0,3} \cong K\left(F_{2}, 1\right)$, a map PConf ${ }_{3}\left(S^{2}\right) \rightarrow S_{0,3}$ up to homotopy is determined by $\operatorname{Hom}\left(P B_{3}\left(S^{2}\right), F_{2}\right)$ up to conjugation. However $P B_{3}\left(S^{2}\right)=P B_{2} / Z=1$ implying that $\operatorname{Hom}\left(P B_{3}\left(S^{2}\right), F_{2}\right)=1$. Therefore, there is a unique section up to homotopy. For the unordered 
case $F_{3}\left(S^{2}\right)$, let $\operatorname{Mod}\left(S_{0,3,1}\right)$ be the mapping class group of $S^{2}$ fixing a set of 3 points and a set of 1 point. There is an exact sequence

$$
1 \rightarrow \operatorname{PMod}\left(S_{0,4}\right) \rightarrow \operatorname{Mod}\left(S_{0,3,1}\right) \rightarrow \Sigma_{3} \rightarrow 1
$$

Since $\pi_{1}\left(\operatorname{PConf}_{3}\left(S^{2}\right) / \Sigma_{3}\right) \cong \Sigma_{3}$ and $\pi_{1}\left(S_{0,3}\right) \cong \operatorname{PMod}\left(S_{0,4}\right)$, we have that $\operatorname{Mod}\left(S_{0,3,1}\right)=\pi_{1}\left(\operatorname{PConf}_{4}\left(S^{2}\right) / \Sigma_{3}\right)$. Therefore, the section of $F_{3}\left(S^{2}\right)$ is the determined by the splittings of the exact sequence (5.4).

Let $\overline{\operatorname{Diff}}\left(S_{0,3,1}\right)$ be the orientation-preserving diffeomorphism group of $S^{2}$ fixing a set of 3 points and a set of 1 point. By definition there is a map $\rho: \overline{\operatorname{Diff}}\left(S_{0,3,1}\right) \rightarrow \operatorname{Mod}\left(S_{0,3,1}\right)$ which induces isomorphism on $\pi_{0}$. A version of the Nielsen Realisation Theorem (e.g. [FM12, Theorem 7.2] and [Wol87]) tells us that a finite subgroup of $\operatorname{Mod}\left(S_{0,3,1}\right)$ has a lift to $\overline{\operatorname{Diff}}\left(S_{0,3,1}\right)$. However every finite subgroup of $\overline{\operatorname{Diff}}\left(S_{0,3,1}\right)$ is cyclic because $\overline{\operatorname{Diff}}\left(S_{0,3,1}\right)$ fixes a point. Therefore every finite subgroup of $\operatorname{Mod}\left(S_{0,3,1}\right)$ is cyclic. Since $\Sigma_{3}$ is noncyclic, (5.4) does not split.

For the special cases $n=4$, we have the following classification.

Theorem 5.6 (Classification of sections of $f_{4}\left(S^{2}\right)$ ). The sections of fiber bundle $f_{4}\left(S^{2}\right)$ correspond to the splittings of the exact sequence

$$
1 \rightarrow F_{3} \rightarrow P B_{5}\left(S^{2}\right) \stackrel{f_{4}\left(S^{2}\right)_{*}}{\longrightarrow} F_{2} \rightarrow 1
$$

up to conjugation.

Proof. We have the following Birman exact sequence; see [FM12, Theorem 4.6].

$$
1 \rightarrow \pi_{1}\left(S_{0,3}\right) \rightarrow P B_{4}\left(S^{2}\right) \stackrel{f_{3}\left(S^{2}\right)_{*}}{\longrightarrow} P B_{3}\left(S^{2}\right) \rightarrow 1 .
$$

Since $P B_{3}\left(S^{2}\right)=1$, we have that $P B_{4}\left(S^{2}\right)=\pi_{1}\left(S_{0,3}\right) \cong F_{2}$. By Proposition 5.4 , the sections of $f_{4}\left(S^{2}\right)$ is determined by the splittings of the following Birman exact sequence up to conjugaction.

$$
1 \rightarrow \pi_{1}\left(S_{0,4}\right) \rightarrow P B_{5}\left(S^{2}\right) \stackrel{f_{4}\left(S^{2}\right)_{*}}{\longrightarrow} P B_{4}\left(S^{2}\right) \rightarrow 1 .
$$

\section{The Case when $S=S_{g}$ a Closed surface of genus $g>1$}

In this section, we prove Theorem 1.1(3). Let $S_{g}^{n}$ be the product of $n$ copies of $S_{g}$. There is a natural embedding $\operatorname{PConf}_{n}\left(S_{g}\right) \subset S_{g}^{n}$. Let $p_{i}: \operatorname{PConf}_{n}\left(S_{g}\right) \rightarrow S_{g}$ be the projection onto the $i$ th component. Denote by $\triangle_{i j} \approx S_{g}^{n-1} \subset S_{g}^{n}$ the $i j$ th diagonal subspace of $S_{g}^{n}$; i.e., $\triangle_{i j}$ consists of points in $S_{g}^{n}$ such that the $i$ th and $j$ th coordinates are equal. Let $H_{i}:=p_{i}^{*} H^{1}\left(S_{g} ; \mathbb{Q}\right)$ and let $\left[S_{g}\right]$ be the fundamental class in $H^{2}\left(S_{g} ; \mathbb{Q}\right)$. Now, we display the computation of $H^{*}\left(\operatorname{PConf}_{n}\left(S_{g}\right) ; \mathbb{Q}\right)$ from Che16.

Lemma 6.1. (1) For $g>1$ and $n>0$,

$$
H^{1}\left(\operatorname{PConf}_{n}\left(S_{g}\right) ; \mathbb{Q}\right) \cong H^{1}\left(S_{g}^{n} ; \mathbb{Q}\right) \cong \bigoplus_{i=1}^{n} H_{i}
$$

(2) We have an exact sequence

$$
1 \rightarrow \oplus_{1 \leq i<j \leq n} \mathbb{Q}\left[G_{i j}\right] \stackrel{\phi}{\rightarrow} H^{2}\left(S_{g}^{n} ; \mathbb{Q}\right) \cong \bigoplus_{i=1}^{n} \mathbb{Q} p_{i}^{*}\left[S_{g}\right] \oplus \bigoplus_{i \neq j} H_{i} \otimes H_{j} \stackrel{P r}{\rightarrow} H^{2}\left(\operatorname{PConf} f_{n}\left(S_{g}\right) ; \mathbb{Q}\right),
$$


where $\phi\left(G_{i j}\right)=\left[\triangle_{i j}\right] \in H^{2}\left(S_{g}^{n} ; \mathbb{Q}\right)$ is the Poincaré dual of the diagonal $\triangle_{i j}$.

Proof. See [Che16, Lemma 3.1].

Let $\left\{a_{k}, b_{k}\right\}_{k=1}^{g}$ be a symplectic basis for $H^{1}\left(S_{g} ; \mathbb{Q}\right)$. For $1 \leq i, j \leq m$, we denote

$$
M_{i, j}=\sum_{k=1}^{n} p_{i}^{*} a_{k} \otimes p_{j}^{*} b_{k}-p_{i}^{*} b_{k} \otimes p_{j}^{*} a_{k} .
$$

Lemma 6.2. The diagonal element $\left[\triangle_{i j}\right]=p_{i}^{*}\left[S_{g}\right]+p_{j}^{*}\left[S_{g}\right]+M_{i j} \in \bigoplus_{i=1}^{n} \mathbb{Q} p_{i}^{*}\left[S_{g}\right] \oplus \bigoplus_{i \neq j} H_{i} \otimes H_{j} \cong$ $H^{2}\left(S_{g}^{n} ; \mathbb{Q}\right)$.

Proof. See [Che16, Lemma 3.2].

The following lemma is the classification of homomorphisms $\pi_{1}\left(\operatorname{PConf}_{n}\left(S_{g}\right)\right) \rightarrow \pi_{1}\left(S_{g}\right)$ from Che16].

Theorem 6.3 (The classification of homomorphisms $\left.\pi_{1}\left(\operatorname{PConf}_{n}\left(S_{g}\right)\right) \rightarrow \pi_{1}\left(S_{g}\right)\right)$. Let $g>1$ and $n>0$. Let $R: \pi_{1}\left(P \operatorname{Conf}_{n}\left(S_{g}\right)\right) \rightarrow \pi_{1}\left(S_{g}\right)$ be a homomorphism. The followings hold:

(1)If $R$ is surjective, then $R=A \circ p_{i *}$ for some $i$ and $A$ an automorphism of $\pi_{1}\left(S_{g}\right)$.

(2)If Image $(R)$ is not a cyclic group, the homomorphism $\pi_{1}\left(P C o n f_{n}\left(S_{g}\right)\right) \rightarrow \pi_{1}\left(S_{g}\right)$ factors through $p_{i *}$ for some $i$.

Proof. See [Che16, Theorem 1.5].

Now, we are ready to prove (3) of Theorem 1.1 .

Proof of (3) of Theorem 1.1. Suppose that there is a map $s: \operatorname{PConf}_{n}\left(S_{g}\right) \rightarrow \operatorname{PConf}_{n+1}\left(S_{g}\right)$ such that $f_{n}\left(S_{g}\right) \circ s=$ identity. Then after post-composing with a forgetful map of the last coordinate, we obtain a map $f: \operatorname{PConf}_{n}\left(S_{g}\right) \rightarrow S_{g}$. We denote

$$
g_{i}: \operatorname{PConf}_{n}\left(S_{g}\right) \stackrel{\left(f, p_{i}\right)}{\longrightarrow} \operatorname{PConf}_{2}\left(S_{g}\right) \subset S_{g} \times S_{g}
$$

Let $\triangle \subset S_{g} \times S_{g}$ be the diagonal subspace and $[\triangle] \in H^{2}\left(S_{g} \times S_{g} ; \mathbb{Q}\right)$ be the Poincaré dual of $\triangle$. Let $f^{*}: H^{1}\left(S_{g}\right) \rightarrow H^{1}\left(\mathrm{PConf}_{n}\left(S_{g}\right)\right)$ and $f_{*}: \pi_{1}\left(\operatorname{PConf}_{n}\left(S_{g}\right)\right) \rightarrow \pi_{1}\left(S_{g}\right)$ be the induced map on cohomology and the fundamental groups. By Lemma 6.3, either $f_{*}$ factors though a forgetful map $p_{i *}$ or Image $\left(f_{*}\right) \cong \mathbb{Z}$. We break the proof into two cases according to the image of $f_{*}$.

Case 1: $\operatorname{Image}\left(f_{*}\right) \cong \mathbb{Z}$. There are two subcases:

(1) If $f^{*}=0$, then $g_{i}^{*}([\triangle])=p_{i}^{*}\left[S_{g}\right] \neq 0$. This contradicts the fact that the image of $g_{i}$ misses $\triangle$.

(2) If $f^{*} \neq 0$, then $\operatorname{Im} f^{*} \cong \mathbb{Z}$ because $f_{*}$ has image $\mathbb{Z}$ on the fundamental groups. We assume that there exists a symplectic basis $\left\{a_{k}, b_{k}\right\}_{k=1}^{g}$ for $H^{1}\left(S_{g} ; \mathbb{Q}\right)$ such that $f^{*}\left(a_{i}\right)=0$ for any $i \neq 1$ and 
$f^{*}\left(b_{i}\right)=0$ for any $i$. Let $f^{*}\left(a_{1}\right)=\left(x_{1}, x_{2}, \ldots, x_{n}\right) \neq 0 \in \bigoplus_{i=1}^{n} H_{i} \cong H^{1}\left(\operatorname{PConf}_{n}\left(S_{g}\right) ; \mathbb{Q}\right)$. Assume without loss of generality that $x_{1} \neq 0$. Therefore for $k \neq 1$ by Lemma 6.2 , we have that

$$
g_{k}^{*}([\triangle])=p_{k}^{*}\left[S_{g}\right]+\sum_{i=1, i \neq k}^{n} x_{i} \smile p_{k}^{*} b_{1} \in \bigoplus_{i=1}^{n} \mathbb{Q} p_{i}^{*}\left[S_{g}\right] \oplus \bigoplus_{i \neq j} H_{i} \otimes H_{j} \cong H^{2}\left(S_{g}^{n} ; \mathbb{Q}\right) .
$$

The coordinate $x_{1} \otimes p_{k}^{*} b_{1}$ is not zero, therefore $g_{k}^{*}([\triangle]) \neq 0$. This contradicts the fact that the image of $g_{i}$ misses $\triangle$.

Case 2: $\boldsymbol{f}_{*}$ factors though the forgetful map $\boldsymbol{p}_{\boldsymbol{i} \text { * }}$. Without loss of generality, we assume that $i=1$. We have that

$$
g_{2}^{*}([\triangle])=f^{*}\left[S_{g}\right]+p_{2}^{*}\left[S_{g}\right]+\sum_{k} f^{*} a_{k} \smile p_{2}^{*} b_{k}-f^{*} b_{k} \smile p_{2}^{*} a_{k} .
$$

Since Image $\left(f^{*}\right) \subset \operatorname{Image}\left(p_{1}^{*}\right)$, we have that $g_{2}^{*}([\triangle])$ only has nonzero terms in $\mathbb{Q} G_{12} \oplus H_{1} \otimes H_{2}$. The fact that $g_{2}$ misses $\triangle$ implies

$$
f^{*}\left[S_{g}\right]+p_{2}^{*}\left[S_{g}\right]+\sum_{k} f^{*} a_{k} \otimes p_{2}^{*} b_{k}-f^{*} b_{k} \otimes p_{2}^{*} a_{k}=\lambda\left(\left[\triangle_{12}\right]\right) \in \mathbb{Q} p_{1}^{*}\left[S_{g}\right] \oplus \mathbb{Q} p_{2}^{*}\left[S_{g}\right] \oplus H_{1} \otimes H_{2} .
$$

The coefficient of $p_{2}^{*}\left[S_{g}\right]$ tells us that $\lambda=1$. Therefore we have that $f^{*}\left[S_{g}\right]=p_{1}^{*}\left[S_{g}\right]$ and

$$
\sum_{k}\left(f^{*} a_{k}-p_{1}^{*} a_{k}\right) \otimes p_{2}^{*} b_{k}-\left(f^{*} b_{k}-p_{1}^{*} b_{k}\right) \otimes p_{2}^{*} a_{k}=0 \in H_{1} \otimes H_{2}
$$

By the property of tensor product, we know that $f^{*} a_{k}-p_{1}^{*} a_{k}=0$ and $f^{*} b_{k}-p_{1}^{*} b_{k}=0$. However in this case, if we look at the map $g_{1}: \operatorname{PConf}_{n}\left(S_{g}\right) \stackrel{\left(f, p_{1}\right)}{\longrightarrow} S_{g} \times S_{g}$. We have that

$g_{1}^{*}([\triangle])=f^{*}\left[S_{g}\right]+p_{1}^{*}\left[S_{g}\right]+\sum_{k} f^{*} a_{k} \smile p_{1}^{*} b_{k}-f^{*} b_{k} \smile p_{1}^{*} a_{k}=2 p_{1}^{*}\left[S_{g}\right]-2 g p_{1}^{*}\left[S_{g}\right]=(2-2 g) p_{1}^{*}\left[S_{g}\right] \neq 0$.

This contradicts the fact that the image of $g_{1}$ misses $\triangle$.

\section{FURTHER QUESTIONS}

In this section we list a few further questions. Let $m, n$ be two positive integers. Let $\left(x_{1}, \ldots, x_{n}\right) \in$ $\operatorname{PConf}_{n}(S)$ for any manifold $S$. Let the permutation group $\Sigma_{m}$ acts on $\operatorname{PConf}_{n+m}(S)$ by permuting the last $m$ points. We have the following fiber bundle:

$$
\operatorname{PConf}_{m}\left(S-\left\{x_{1}, \ldots, x_{n}\right\}\right) / \Sigma_{m} \rightarrow \operatorname{PConf}_{n+m}(S) / \Sigma_{m} \stackrel{f_{n+m, n}(S)}{\longrightarrow} \operatorname{PConf}_{n}(S) .
$$

Here denote by $f_{n+m, n}(S)$ the forgetful map that forgets the first $n$ points. A section of the fiber bundle (7.1) is called a multi-section.

Problem 7.1. Classify the continuous sections of the fiber bundle (7.1) up to homotopy for $S$ a surface.

Problem 7.2. Classify the continuous sections of the fiber bundle (7.1) up to homotopy for any manifold $S$. 


\section{REFERENCES}

[BCWW06] A. J. Berrick, F. R. Cohen, Y. L. Wong, and J. Wu. Configurations, braids, and homotopy groups. J. Amer. Math. Soc., 19(2):265-326, 2006.

[BLM83] J. Birman, A. Lubotzky, and J. McCarthy. Abelian and solvable subgroups of the mapping class groups. Duke Math. J., 50(4):1107-1120, 1983.

[BM06] R. Bell and D. Margalit. Braid groups and the co-Hopfian property. J. Algebra, 303(1):275-294, 2006.

[Cas16] F. Castel. Geometric representations of the braid groups. Astérisque, (378):vi+175, 2016.

[Che16] L. Chen. The universal $n$-pointed surface bundle only has $n$ sections. Pre-print, https://arxiv.org/abs/1611.04624, 2016.

[CP15] F. Cantero and M. Palmer. On homological stability for configuration spaces on closed background manifolds. Doc. Math., 20:753-805, 2015.

[EE69] C. Earle and J. Eells. A fibre bundle description of Teichmüller theory. J. Differential Geometry, 3:19-43, 1969.

[EWG15] J. Ellenberg and J. Wiltshire-Gordon. Algebraic structures on cohomology of configuration spaces of manifolds with flows. Pre-print, https://arxiv.org/pdf/1508.02430.pdf, 2015 .

[Fad62] E. Fadell. Homotopy groups of configuration spaces and the string problem of Dirac. Duke Math. J., 29:231-242, 1962.

[FH01] E. Fadell and S. Husseini. Geometry and topology of configuration spaces. Springer Monographs in Mathematics. Springer-Verlag, Berlin, 2001.

[FLP12] A. Fathi, F. Laudenbach, and V. Poénaru. Thurston's work on surfaces, volume 48 of Mathematical Notes. Princeton University Press, Princeton, NJ, 2012. Translated from the 1979 French original by Djun M. Kim and Dan Margalit.

[FM12] B. Farb and D. Margalit. A primer on mapping class groups, volume 49 of Princeton Mathematical Series. Princeton University Press, Princeton, NJ, 2012.

[FN62] E. Fadell and L. Neuwirth. Configuration spaces. Math. Scand., 10:111-118, 1962.

[GG03] D. L. Gonçalves and J. Guaschi. On the structure of surface pure braid groups. J. Pure Appl. Algebra, 182(1):33-64, 2003.

[GG05] D. L. Gonçalves and J. Guaschi. The braid group $B_{n, m}\left(\mathbb{S}^{2}\right)$ and a generalisation of the Fadell-Neuwirth short exact sequence. J. Knot Theory Ramifications, 14(3):375-403, 2005.

[HT02] H. Hamidi-Tehrani. Groups generated by positive multi-twists and the fake lantern problem. Algebr. Geom. Topol., 2:1155-1178, 2002.

[Mar02] D. Margalit. A lantern lemma. Algebr. Geom. Topol., 2:1179-1195, 2002.

[McC82] J. McCarthy. Normalizers and centralizers of pseudo-anosov mapping classes. Preprint: http://users.math.msu.edu/users/mccarthy/publications/normcent.pdf, 1982.

[McD75] D. McDuff. Configuration spaces of positive and negative particles. Topology, 14:91$107,1975$. 
[MM09] D. Margalit and J. McCammond. Geometric presentations for the pure braid group. J. Knot Theory Ramifications, 18(1):1-20, 2009.

[Seg74] G. Segal. Categories and cohomology theories. Topology, 13:293-312, 1974.

[Sma59] S. Smale. Diffeomorphisms of the 2-sphere. Proc. Amer. Math. Soc., 10:621-626, 1959.

[Tot96] B. Totaro. Configuration spaces of algebraic varieties. Topology, 35(4):1057-1067, 1996.

[Wol87] S. Wolpert. Geodesic length functions and the Nielsen problem. J. Differential Geom., 25(2):275-296, 1987.

California Institute of Technology

Department of Mathematics

Pasadena, CA 91125, USA

E-mail: chenlei1991919@gmail.com 